\title{
Theoretical-experimental analysis of heat transfer in nonhomogeneous solids via improved lumped formulation, integral transforms and infrared thermography
}

\author{
Diego C. Knupp, Carolina P. Naveira-Cotta, João V.C. Ayres, Renato M. Cotta*, Helcio R.B. Orlande \\ Laboratory of Transmission and Technology of Heat, LTTC, Mechanical Engineering Dept. - POLI E COPPE, UFRJ, Universidade Federal do Rio de Janeiro, Cidade Universitária, \\ Cx. Postal 68503, Rio de Janeiro, RJ, CEP 21945-970, Brazil
}

\section{A R T I C L E I N F O}

Article history:

Received 30 August 2011

Received in revised form

11 January 2012

Accepted 13 January 2012

Available online 27 February 2012

\section{Keywords:}

Heat conduction

Heterogeneous media

Lumped-differential analysis

Infrared thermography

Integral transforms

Bayesian inference

Inverse problems

\begin{abstract}
A B S T R A C T
Theoretical and experimental methodologies for the identification of spatially variable thermophysical properties and for simulating multidimensional heat transfer in heterogeneous materials are illustrated by using plate samples with aluminum oxide nanoparticles dispersed in a polymeric matrix. First, the heterogeneous nanocomposite plate is thermally characterized by means of a fairly simple experimental setup which can be modeled by a one-dimensional heat conduction formulation with space variable properties. Non-intrusive temperature measurements are obtained via infrared thermography, while the direct problem is handled by an error-controlled integral transform solution with an improved lumpeddifferential formulation, and the inverse analysis is undertaken via Bayesian inference, making use of the Markov Chain Monte Carlo method. Then, in order to illustrate the application of the methodologies here presented, an experimental multidimensional demonstration is provided consisting of a small electrical resistance attached to the plate, simulating a heat generating electronic device installed on the nanocomposite substrate, which in such situation works as a heat spreader modeled by an improved lumpeddifferential two-dimensional heat conduction formulation. The integral transform solution of the lumped-differential two-dimensional problem is then critically compared against the infrared thermography experimental results.
\end{abstract}

(c) 2012 Elsevier Masson SAS. All rights reserved.

\section{Introduction}

Nonhomogeneous materials morphology and composition present endless combinations and arrangements due to design and manufacturing, such as in the case of nanocomposites, tailored to meet a variety of property and performance requirements [1]. Thus, the characterization of their physical properties is to be made on a case by case basis, either in the form of averaged effective properties or as spatially variable functions for improved accuracy [2]. In the determination of local variations in thermophysical properties within heterogeneous solids, one must employ an experimental technique that provides a sufficient amount of information on spatially distributed temperature measurements, in order to provide a firm basis for application of the appropriate inverse problem analysis, such as the non-intrusive infrared thermography [3-6].

\footnotetext{
* Corresponding author. Tel: +55 212562 8567; fax: +55 2125628383.

E-mail addresses: cotta@mecanica.coppe.ufrj.br, renatocotta@hotmail.com (R.M. Cotta).
}

Inversion methods able to process such measured fields and estimate the corresponding parameter maps, are defined by both a direct model and an estimation procedure [7]. In general, such inverse methods require accurate and computationally fast direct problem solution methodologies, so as to handle the large amount of data that might be available and to allow for the computationally intensive iterative analysis often required by the inverse problem solution. Among the most adequate direct problem solution techniques for inverse analysis, we may place the Generalized Integral Transform Technique (GITT) for the hybrid numerical-analytical solution of convection-diffusion problems [8-10]. This approach is based on extending the classical integral transform method making it sufficiently flexible to handle problems that are not a priori transformable, such as in the case of problems with arbitrarily space-dependent and nonlinear coefficients in either the equation or the boundary conditions. This class of hybrid numerical-analytical methods was recently employed in the direct and inverse analysis of heat conduction in heterogeneous media [11-15], including a novel proposition of working the inverse analysis on the transformed field, upon integral transformation of the experimental data $[13,14]$. In addition, the effort to integrate the 


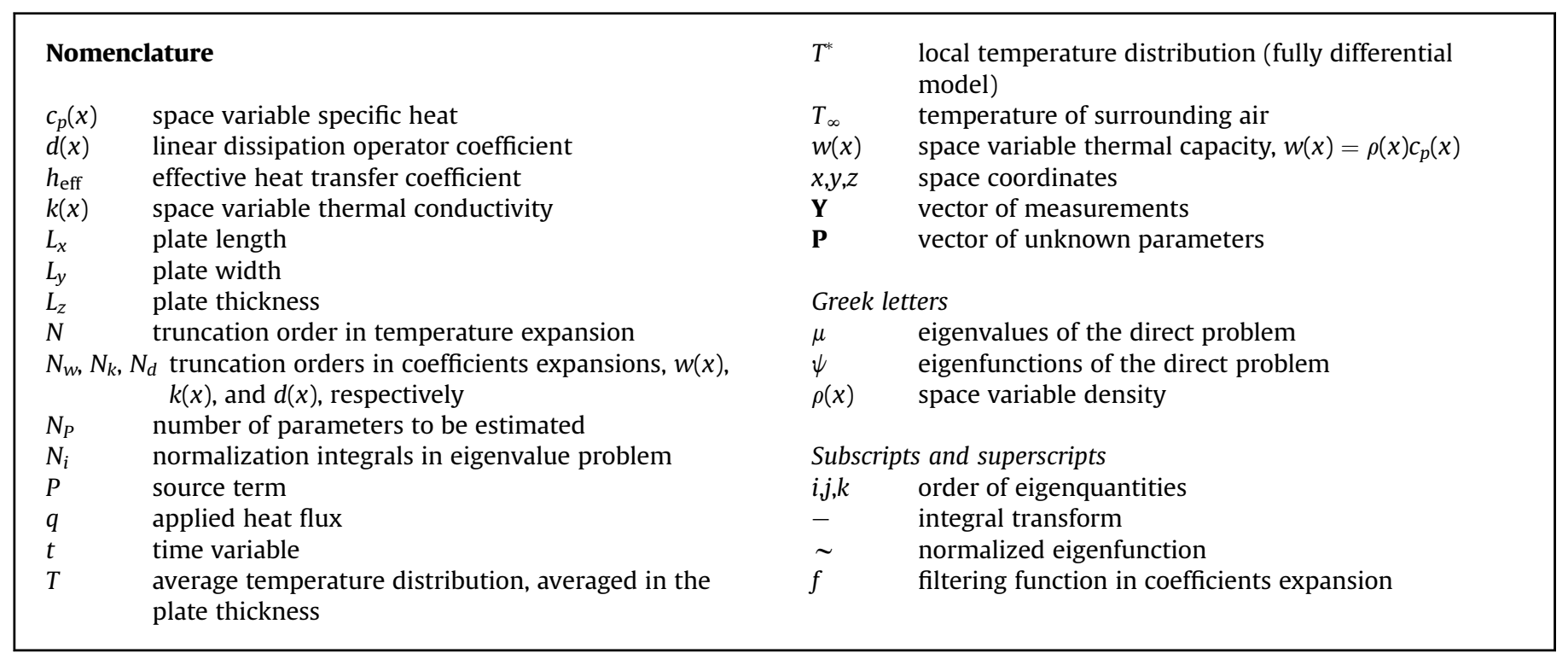

knowledge on the GITT application into a general purpose computational code resulted in a recently developed open source mixed symbolic-numerical code called UNIT (UNified Integral Transforms) [16,17], an algorithm implementation and development platform for researchers and engineers interested on hybrid integral transform solutions of convection-diffusion problems.

In this context, we here present results for an experiment involving a partially heated plate of a nanocomposite system with space-dependent thermophysical properties composed of polyester resin as the matrix material and alumina nanoparticles as filler. First, the nanocomposite plate is thermally characterized by means of a quite simple experimental setup which can be modeled by a one-dimensional heat conduction formulation, employing a heater of the same width as the sample.

A novel feature of the approach herein presented with respect to previous contributions is the proposition of an improved lumpeddifferential formulation [9], averaged across the plate's thickness, instead of the classical lumped analysis, offering an enhanced approximation for the temperatures at the exposed surface of the plate, which are used for the solution of the inverse problem and direct comparison with the infrared thermography measurements. This improved formulation to a certain extent eliminates the limitation of having a thermally thin plate sample, by taking into account, though approximately, the temperature gradients across the sample thickness.

Then, in order to illustrate the application of these methodologies, a small electrical resistance is attached to the nanocomposite substrate, which in such situation works as a heat spreader for an electronic device, and requiring an improved two-dimensional lumped-differential heat conduction model for its analysis. Temperature measurements are again obtained via infrared thermography.

The infrared camera employed was the SC660 model from Flir Systems Inc, with dedicated data handling routines for quantitative thermographic analysis. The inverse analysis procedure employs both the infrared thermography for data acquisition and the integral transform approach for the direct problem solution [13,14], implemented on the Mathematica 7.0 platform [18]. Based on Bayesian inference [19-21], the methodology advanced in [13,14] introduces the use of integral transformed temperature measurements and the estimation within the transformed domain, providing a pronounced reduction on experimental data handling and computational effort.
Therefore, the present work provides both a verification of the variable thermophysical properties estimation methodology as well as its application in the multidimensional heat transfer analysis of nonhomogeneous nanocomposite heat spreaders.

\section{Experimental setup}

The experimental setup presented in Fig. 1 employs temperature measurements obtained from the infrared camera FLIR SC660, a high performance infrared system with $640 \times 480$ image resolution, and $-40{ }^{\circ} \mathrm{C}$ to $1500{ }^{\circ} \mathrm{C}$ temperature range. The main components of the setup are marked on Fig. 1a as (a) IR camera (FLIR SC660); (b) camera stand for vertical experiment configuration; (c) frame with the sandwich nanocomposite plate-heater-thermal insulation; (d) sample support; (e) data acquisition system (Agilent 34970-A); (f) microcomputer for data acquisition. Fig. 1b shows the nanocomposite plate used in this experiment, which is composed by polyester resin as matrix and alumina nanoparticles as filler, manufactured in such a way that roughly $3 / 4$ of the plate's length has $28.5 \%$ of alumina nanoparticles in mass and the other $1 / 4$ of the plate's length is composed only by polyester resin, with no addition of filler. The plate's thickness is $1.51 \mathrm{~mm}$ and its lateral and vertical dimensions are $40 \times 80 \mathrm{~mm}$. An electrical resistance $(38.2 \Omega$ ) was employed in the heating of the plate at their contacting interface, with the same lateral dimensions as of the plate but half the length $(40 \times 40 \mathrm{~mm})$, here joined at the upper half of the plate's height. The resistance is attached to the nanocomposite plate with the aid of a thermal compound paste, being the opposite face insulated and kept in place by the frame, with insulated corners. In order to illustrate an application of the methodologies here presented, we also show some results in which the $40 \times 40 \mathrm{~mm}$ electrical resistance is substituted by a smaller one, with $14 \times 14 \mathrm{~mm}$ lateral dimensions and $29.8 \Omega$, simulating an electronic chip installed on the nanocomposite substrate, which in such situation works as a heat spreader for the electronic device. In such case the heat diffusion along the plate's width cannot be neglected and we consider a two-dimensional lumped-differential model for the problem formulation. In Fig. 2 the experimental setups are schematically represented.

In order to reduce uncertainty in the IR camera readings, the plate surface that faces the infrared camera was painted with a graphite ink, which brought its emissivity to around $\varepsilon=0.97$, as stated by the ink manufacturer. 
a

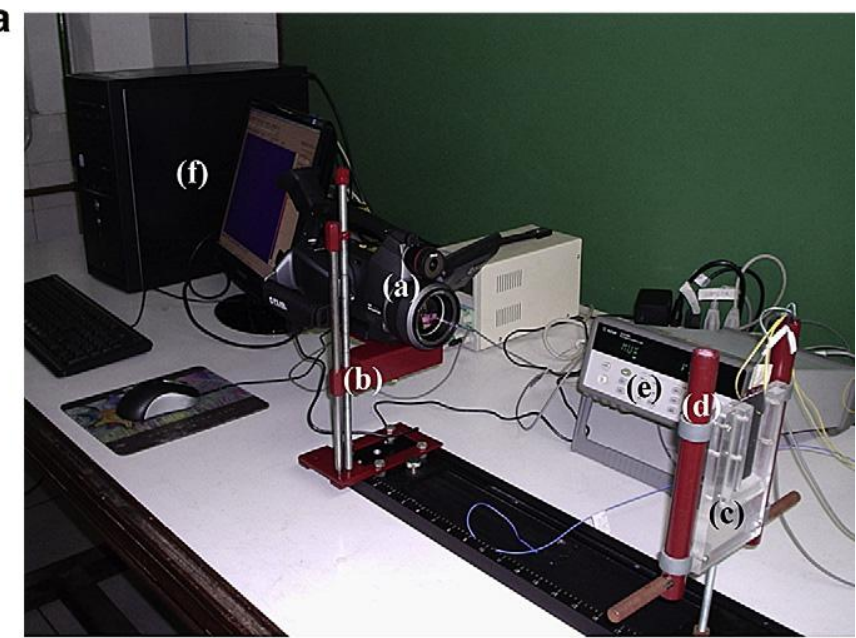

b

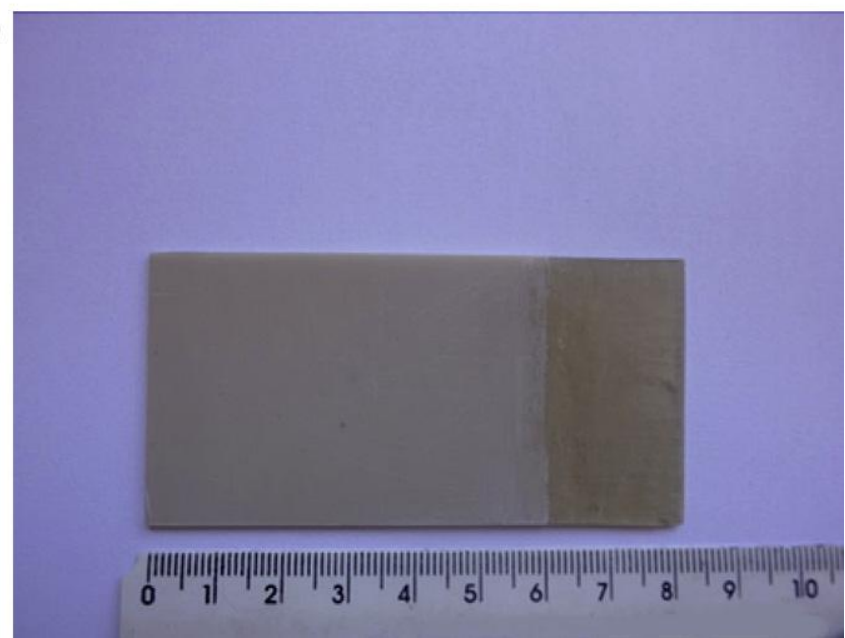

Fig. 1. (a) General view of the experimental setup for the infrared thermography analysis. (b) Polyester resin-alumina nanocomposite plate used in the experiment with dimensions $1.51 \mathrm{~mm}$ (thickness), $40 \mathrm{~mm}$ (width) and $80 \mathrm{~mm}$ (length).

The experimental procedure is initiated by prescribing a voltage difference to be imposed on the electrical resistance, with a DC voltage regulator. The data acquisition is started and after a certain number of preliminary measurements to allow for averaging the initial conditions, the DC source switch is turned on to heat the plate (a nominal voltage of $8 \mathrm{~V}$ and $3.6 \mathrm{~V}$ has been applied through the DC source for the experiments, respectively, using the $40 \times 40 \mathrm{~mm}$ and $14 \times 14 \mathrm{~mm}$ electrical resistances). The temperature increase may be followed through the computer monitoring. Figs. 3 illustrate the images produced by the FLIR SC660 camera after some elapsed heating time both for the case with the $40 \times 40 \mathrm{~mm}$ electrical resistance, at $t=200 \mathrm{~s}$, in Fig. 3a, and for the case with the $14 \times 14 \mathrm{~mm}$ electrical resistance, at $t=100 \mathrm{~s}$, in Fig. 3b. Once steady state is achieved, the DC source is turned off.

\section{Problem formulation and solution methodology}

Consider a plate subjected to a prescribed heat flux at one face and convective and radiative heat losses at the opposite face (see Fig. 4), which represents the physical phenomena that takes place in the experiment with the heating element of the same width as the sample plate $(40 \times 40 \mathrm{~mm})$. Neglecting the temperature gradients on the width direction, this problem can be formulated as:
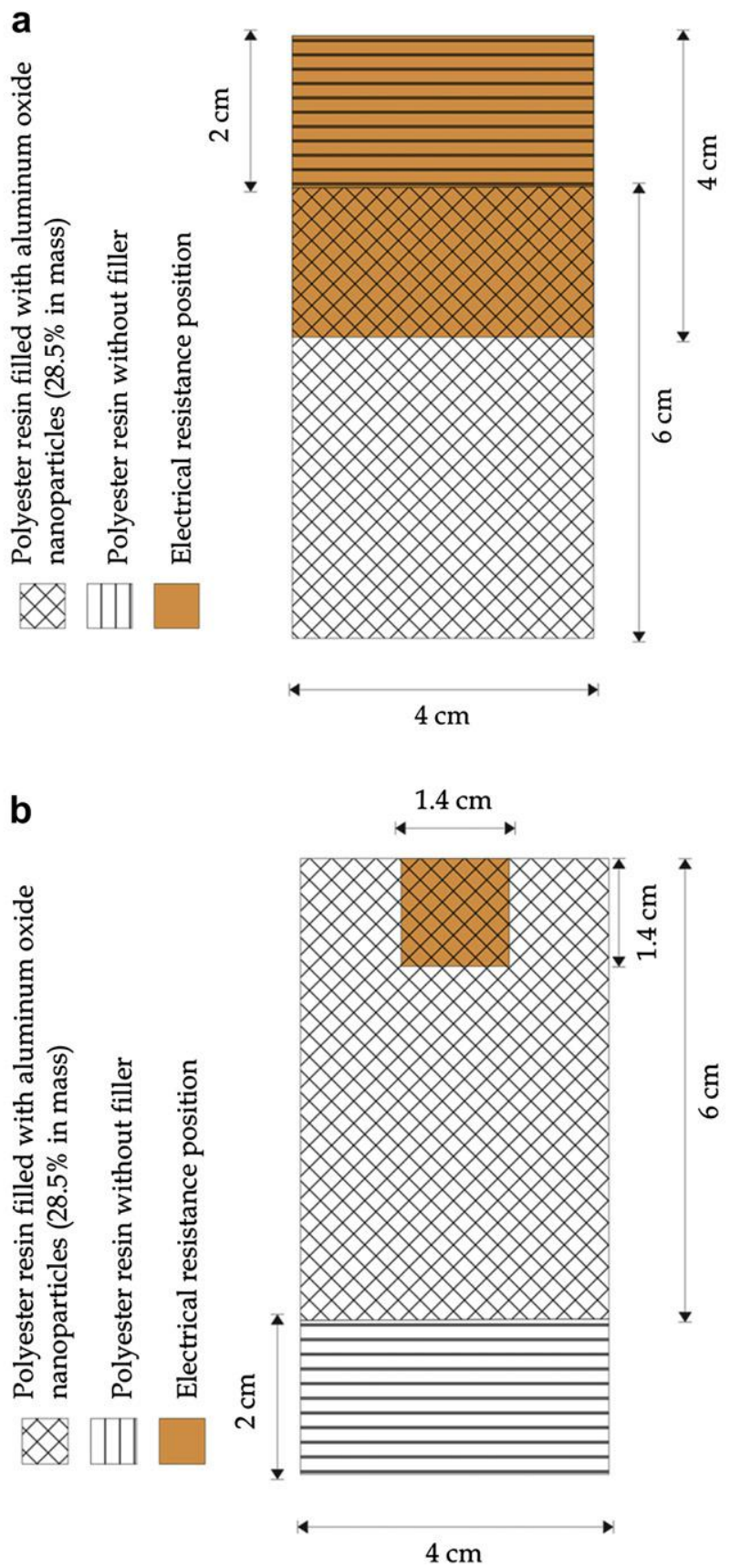

Fig. 2. (a) Schematic representation of the experimental setup used for the inverse analysis procedure. (b) Schematic representation of the experimental setup used to simulate an electronic device installed on the nanocomposite substrate as a heat spreader.

$$
w(x) \frac{\partial T^{*}(x, z, t)}{\partial t}=\frac{\partial}{\partial x}\left(k(x) \frac{\partial T^{*}}{\partial x}\right)+k(x) \frac{\partial^{2} T^{*}}{\partial z^{2}}, \quad 0<x<L_{x},
$$$$
0<z<L_{z}, t>0
$$

$T^{*}(x, z, 0)=T_{\infty}, 0 \leq x \leq L_{x}, 0 \leq z \leq L_{z}$

$\left.\frac{\partial T^{*}}{\partial x}\right|_{x=0}=0,\left.\quad \frac{\partial T^{*}}{\partial x}\right|_{x=L_{x}}=0$ 

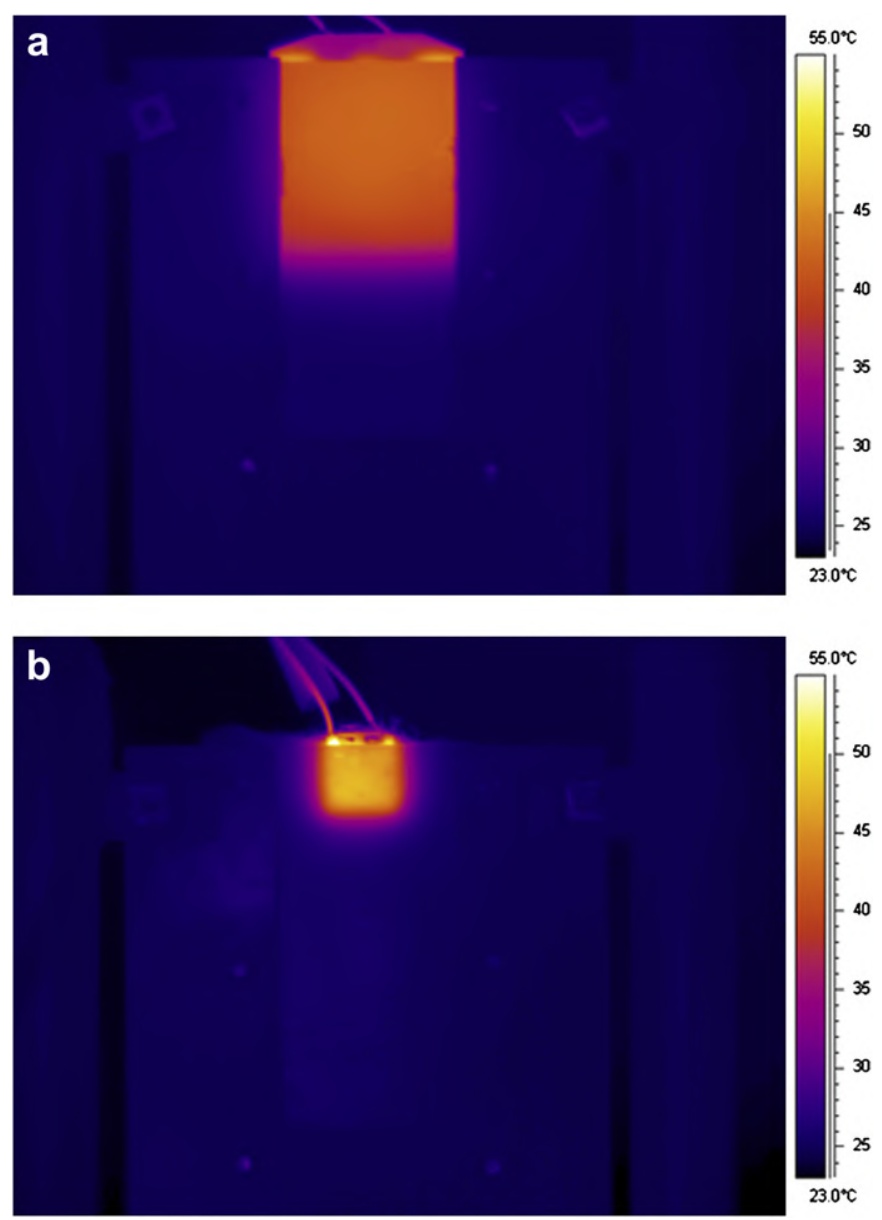

Fig. 3. (a) Infrared camera image acquired at $t=200 \mathrm{~s}$ during heating period for the case with the $40 \times 40 \mathrm{~mm}$ electrical resistance. (b) Infrared camera image acquired at $t=100 \mathrm{~s}$ during heating period for the case with the $14 \times 14 \mathrm{~mm}$ electrical resistance.

$-\left.k(x) \frac{\partial T^{*}}{\partial z}\right|_{z=0}=q(x, t),\left.k(x) \frac{\partial T^{*}}{\partial z}\right|_{z=L_{z}}+h_{\mathrm{eff}}(x) T^{*}=h_{\mathrm{eff}}(x) T_{\infty}$

where the variable thermal capacitance coefficient is given by $w(x)=\rho(x) c_{p}(x)$. Assuming that the plate is thermally thin, we can make use of a straigthforward lumped formulation across the sample thickness, as proposed in $[13,14]$, with the definition of the average temperature across the $z$ direction:

$T(x, t)=\frac{1}{L_{z}} \int_{0}^{L_{z}} T^{*}(x, z, t) d z$

Making use of eq. (2) and the boundary conditions given by eqs. (1e,f), problem (1) can be integrated over the plate thickness and be rewritten as:

$$
\begin{aligned}
w(x) \frac{\partial T(x, t)}{\partial t}= & \frac{\partial}{\partial x}\left(k(x) \frac{\partial T}{\partial x}\right)+\frac{q(x, t)}{L_{z}}+\frac{h_{\mathrm{eff}}(x) T_{\infty}}{L_{z}} \\
& -\frac{h_{\mathrm{eff}}(x) T^{*}\left(x, L_{z}, t\right)}{L_{z}}, 0<x<L_{x}, t>0
\end{aligned}
$$

$T(x, 0)=T_{\infty}, 0 \leq x \leq L_{x}$

$\left.\frac{\partial T}{\partial x}\right|_{x=0}=0,\left.\frac{\partial T}{\partial x}\right|_{x=L_{x}}=0$

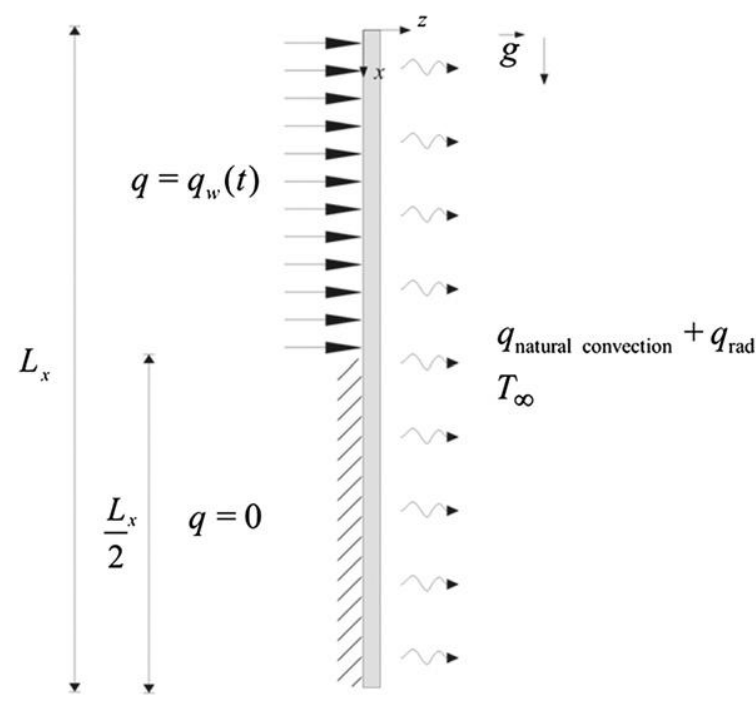

Fig. 4. Schematic representation of the experimental setup for thermophysical properties identification in heterogeneous media.

Thus, the classical lumped approach would simply involve the approximation that the boundary temperatures are very close to the average temperature across the slab thickness, and eq. (3a) would then become a one-dimensional partial differential formulation for the average temperature. Here, however, we seek an improved lumped-differential formulation, in an attempt to offer enhanced approximations for the temperature at the exposed surface of the plate at $z=L_{z}$. The basic idea is to provide a better relation between the boundary and the averaged temperatures, which are to be developed from Hermite-type approximations of the integrals that define the average temperatures and heat fluxes, based on the so called Coupled Integral Equations Approach (CIEA) [9,22-24]. For instance, one may employ Hermite formulas such as the $H_{1,1}$ and $H_{0,0}$ approximations, which are essentially the corrected and plain trapezoidal rules, respectively. When these Hermite formulas are applied to approximate the average temperature and the average heat flux, respectively, one obtains:

$$
\begin{aligned}
T(x, t)= & \frac{1}{L_{z}} \int_{0}^{L_{z}} T^{*}(x, z, t) d z \cong \frac{1}{2}\left[T^{*}(x, z=0, t)+T^{*}\left(x, z=L_{z}, t\right)\right] \\
& +\frac{L_{z}}{12}\left[\left.\frac{\partial T^{*}}{\partial z}\right|_{z=0}-\left.\frac{\partial T^{*}}{\partial z}\right|_{z=L_{z}}\right] \\
\int_{0}^{L_{z}} \frac{\partial T^{*}}{\partial z} d z & =T^{*}\left(x, z=L_{z}, t\right)-T^{*}(x, z=0, t) \\
& \cong \frac{L_{z}}{2}\left[\left.\frac{\partial T^{*}}{\partial z}\right|_{z=0}+\left.\frac{\partial T^{*}}{\partial z}\right|_{z=L_{z}}\right]
\end{aligned}
$$

Eqs. (4a,b) can be solved for $T^{*}\left(x, L_{z}, t\right)$, employing the boundary conditions $(1 \mathrm{e}, \mathrm{f})$ to eliminate the remaining boundary quantities, yielding:

$$
T^{*}\left(x, L_{z}, t\right) \cong \frac{-L_{z} q(x, t)+6 k(x) T(x, t)+2 L_{z} T_{\infty} h_{\mathrm{eff}}(x)}{6 k(x)+2 L_{z} h_{\mathrm{eff}}(x)}
$$

Thus, eq. (4c) provides a more complete relation between the surface temperature and the thickness averaged temperature, which accounts for the non-uniform temperature distribution across the plate thickness. Substituting eq. (4c) into eq. (3a), 
problem (3) can be solved for the averaged potential $T(x, t)$ and improved results for the temperature at the exposed surface of the plate, at $z=L_{z}$, can be readily obtained with the aid of eq. (4c). The resulting improved lumped-differential formulation for the average temperature is given by:

$$
\begin{aligned}
& w(x) \frac{\partial T(x, t)}{\partial t}=\frac{\partial}{\partial x}\left(k(x) \frac{\partial T(x, t)}{\partial x}\right)-d(x) T(x, t)+P(x, t) \\
& 0<x<L_{x}, t>0
\end{aligned} \begin{aligned}
& d(x)=\frac{6 k(x) h_{\mathrm{eff}}(x)}{L_{z}\left[6 k(x)+2 L_{z} h_{\mathrm{eff}}(x)\right]} \\
& P(x, t)=\frac{q(x, t)}{L_{z}}+\frac{q(x, t) h_{\mathrm{eff}}(x)}{6 k(x)+2 L_{z} h_{\mathrm{eff}}(x)}+\frac{h_{\mathrm{eff}}(x) T_{\infty}}{L_{z}} \\
& \quad-\frac{2 T_{\infty} h_{\mathrm{eff}}^{2}(x)}{6 k(x)+2 L_{z} h_{\mathrm{eff}}(x)} \\
& T(x, 0)=T_{\infty}, 0 \leq x \leq L_{x} \\
& \left.\frac{\partial T}{\partial x}\right|_{x=0}=0,\left.\frac{\partial T}{\partial x}\right|_{x=L_{x}}=0
\end{aligned}
$$

The proposed formulation includes the space variable thermal conductivity and thermal capacity, through the coefficients $w(x)$ and $k(x)$, responsible for the information related to the heterogeneity of the medium.

The formal exact solution of problem (5) is obtained with the Classical Integral Transform Method [11], and is written as:

$T(x, t)=T_{\infty}+\sum_{i=1}^{\infty} \tilde{\psi}_{i}(x) \int_{0}^{t} \bar{g}_{i}\left(t^{\prime}\right) e^{-\mu_{i}^{2}\left(t-t^{\prime}\right)} d t^{\prime}$

where the eigenvalues $\mu_{i}$ and eigenfunctions $\psi_{i}(x)$, are obtained from the eigenvalue problem that contains the information about the heterogeneous medium, in the form:

$\frac{d}{d x}\left[k(x) \frac{d \psi_{i}(x)}{d x}\right]+\left(\mu_{i}^{2} w(x)-d(x)\right) \psi_{i}(x)=0, x \in\left[0, L_{x}\right]$

with boundary conditions

$\frac{d \psi_{i}(x)}{d x}=0, x=0, \frac{d \psi_{i}(x)}{d x}=0, x=L_{x}$

Also, the other quantities that appear in the exact solution (6) are computed after solving problem (7), such as

$\tilde{\psi}_{i}(x)=\frac{\psi_{i}(x)}{\sqrt{N_{i}}}$, normalized eigenfunctions

$N_{i}=\int_{0}^{L_{x}} w(x) \psi_{i}^{2}(x) d x$, normalization integrals

$\bar{g}_{i}(t)=\int_{0}^{L_{x}} P(x, t) \tilde{\psi}_{i}(x) d x$, transformed source terms

The Generalized Integral Transform Technique (GITT) [8-10] is here employed in the solution of the Sturm-Liouville problem (Eqs. $7 a-c)$ via the proposition of a simpler auxiliary eigenvalue problem, and expanding the unknown eigenfunctions in terms of the chosen basis $[11,14]$. The variable equation coefficients are themselves expanded in terms of known eigenfunctions [11,14], so as to allow for a fully analytical implementation of the coefficients matrices in the transformed system. For instance, the coefficients $w(x), k(x)$ and $d(x)$ are expanded in terms of eigenfunctions with first kind boundary conditions, together with a filtering solution to enhance convergence, in the following form:

$$
\begin{aligned}
& w(x)=w_{f}(x)+\sum_{k=1}^{\infty} \tilde{\Gamma}_{k}(x) \bar{w}_{k}, \text { inverse } \\
& \bar{w}_{k}=\int_{0}^{L_{x}} w^{*}(x)\left[w(x)-w_{f}(x)\right] \tilde{\Gamma}_{k}(x) d x, \text { transform } \\
& k(x)=k_{f}(x)+\sum_{k=1}^{\infty} \tilde{\Gamma}_{k}(x) \bar{k}_{k}, \text { inverse } \\
& \bar{k}_{k}=\int_{0}^{L_{x}} w^{*}(x)\left[k(x)-k_{f}(x)\right] \tilde{\Gamma}_{k}(x) d x, \text { transform } \\
& d(x)=d_{f}(x)+\sum_{k=1}^{\infty} \tilde{\Gamma}_{k}(x) \bar{d}_{k}, \text { inverse } \\
& \bar{d}_{k}=\int_{0}^{L_{x}} w^{*}(x)\left[d(x)-d_{f}(x)\right] \tilde{\Gamma}_{k}(x) d x, \text { transform }
\end{aligned}
$$

where $w^{*}(x)$ is the weighting function for the chosen normalized eigenfunction $\tilde{\Gamma}_{k}(x)$. The expansion basis may be chosen by employing the same auxiliary problem, but with first order boundary conditions, while the filtering function could be a simple analytic function that satisfies the boundary values for the original coefficients or just an average value of the respective coefficient. This procedure shall also be of interest in the function estimation task, when the transformed coefficients such as in eqs. (9a,c,e) will be the parameters to be estimated.

For the illustration of the methodologies presented in this work, an application is considered in which a small electrical resistance is attached to the heterogeneous plate, simulating an electronic device installed on the nanocomposite substrate, which works as a heat spreader. This problem is schematically represented in Fig. 5a,b and the three-dimensional formulation is given by:

$$
\begin{aligned}
& w(x) \frac{\partial T^{*}(x, y, z, t)}{\partial t}=\frac{\partial}{\partial x}\left(k(x) \frac{\partial T^{*}}{\partial x}\right)+k(x) \frac{\partial^{2} T^{*}}{\partial y^{2}}+k(x) \frac{\partial^{2} T^{*}}{\partial z^{2}}, \\
& 0<x<L_{x}, 0<y<L_{y}, 0<z<L_{z}, t>0
\end{aligned}
$$

$T^{*}(x, y, z, 0)=T_{\infty}, 0 \leq x \leq L_{x}, 0 \leq y \leq L_{y} \quad 0 \leq z \leq L_{z}$

$\left.\frac{\partial T^{*}}{\partial x}\right|_{x=0}=0,\left.\frac{\partial T^{*}}{\partial x}\right|_{x=L_{x}}=0$

$\left.\frac{\partial T^{*}}{\partial y}\right|_{y=0}=0,\left.\frac{\partial T^{*}}{\partial y}\right|_{x=L_{y}}=0$

$-\left.k(x) \frac{\partial T^{*}}{\partial z}\right|_{z=0}=q(x, t),\left.k(x) \frac{\partial T^{*}}{\partial z}\right|_{z=L_{z}}+h_{\mathrm{eff}}(x) T^{*}=h_{\mathrm{eff}}(x) T_{\infty}$

where $w(x)$ and $k(x)$ are again the space-dependent thermal capacity and thermal conductivity, respectively, $h_{\mathrm{eff}}(x, y)$ is the 
a

$\frac{\partial T}{\partial x}=0$

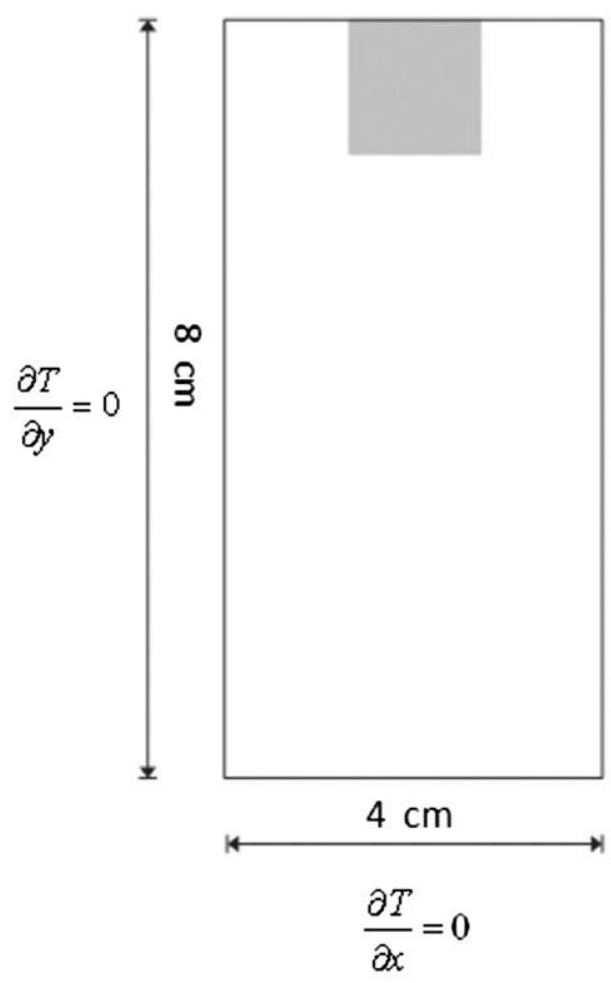

b

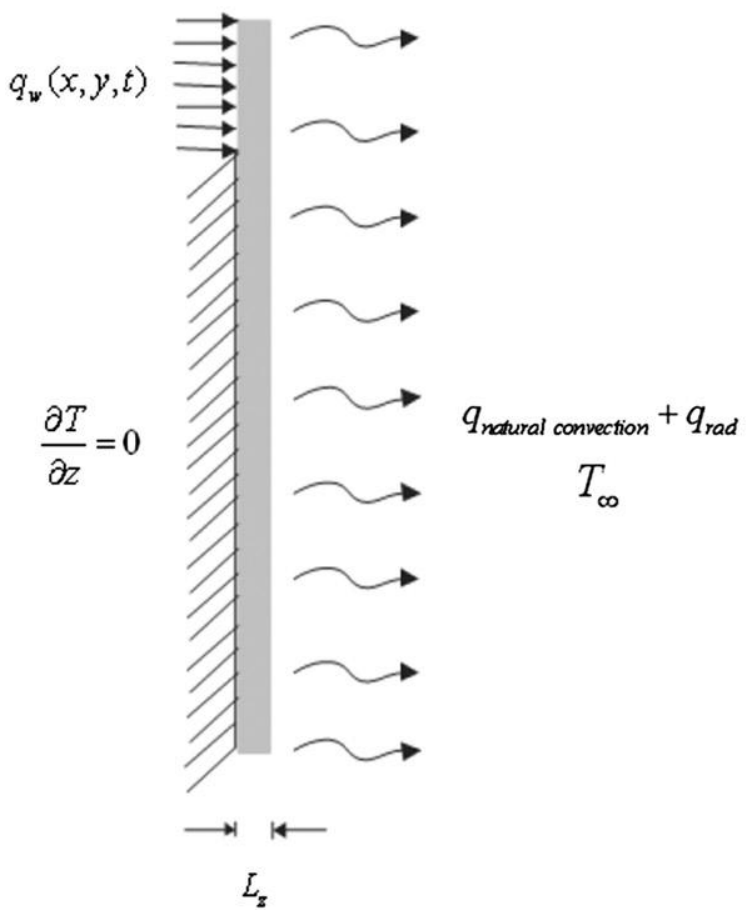

Fig. 5. Schematic representation of the problem used to simulate an electronic device installed on the nanocomposite substrate as a heat spreader: (a) front view. (b) lateral view. effective heat transfer coefficient, $q(x, y, t)$ is the applied heat flux and $L_{x}, L_{y}$ and $L_{z}$ are the plate's length, width and thickness, respectively. After making use of the improved lumped formulation across the plate thickness, the following problem is obtained for the thickness averaged temperature field, $T(x, y, t)$ :

$$
\begin{aligned}
& w(x) \frac{\partial T(x, y, t)}{\partial t}=\frac{\partial}{\partial x}\left(k(x) \frac{\partial T}{\partial x}\right)+k(x) \frac{\partial^{2} T}{\partial y^{2}}+\frac{q_{w}(x, y, t)}{L_{z}} \\
& -\frac{h_{\mathrm{eff}}(x, y)}{L_{z}}\left(T_{s}(x, y, t)-T_{\infty}\right), \\
& 0<x<L_{x}, 0<y<L_{y}, t>0 \\
& T(x, y, 0)=T_{\infty} \\
& \left.\frac{\partial T}{\partial x}\right|_{x=0}=0,\left.\frac{\partial T}{\partial x}\right|_{x=L_{x}}=0 \\
& \left.\frac{\partial T}{\partial y}\right|_{y=0}=0,\left.\frac{\partial T}{\partial y}\right|_{y=L_{y}}=0
\end{aligned}
$$

where $T_{s}(x, y, t)$ should be an enhanced approximation of the temperature at the exposed surface of the plate, which can be written in terms of $T(x, y, t)$ after employing Hermite-type approximations similar to what has been done in eq. (4) above, yielding:

$T_{S}(x, y, t) \cong \frac{-L_{z} q(x, y, t)+6 k(x) T(x, y, t)+2 L_{z} T_{\infty} h_{\mathrm{eff}}(x, y)}{6 k(x)+2 L_{z} h_{\mathrm{eff}}(x, y)}$

The resulting improved lumped-differential formulation for the average temperature $T(x, y, t)$ is thus given by:

$$
\begin{aligned}
& w(x) \frac{\partial T(x, y, t)}{\partial t}=\frac{\partial}{\partial x}\left(k(x) \frac{\partial T}{\partial x}\right)+k(x) \frac{\partial^{2} T}{\partial y^{2}}-d(x, y) T+P(x, y, t), \\
& 0<x<L_{x}, 0<y<L_{y}, t>0 \\
& d(x, y)=\frac{6 k(x) h_{\mathrm{eff}}(x, y)}{L_{z}\left[6 k(x)+2 L_{z} h_{\mathrm{eff}}(x, y)\right]} \\
& P(x, y, t)=\frac{q(x, y, t)}{L_{z}}+\frac{q(x, y, t) h_{\mathrm{eff}}(x, y)}{6 k(x)+2 L_{z} h_{\mathrm{eff}}(x, y)}+\frac{h_{e f f}(x, y) T_{\infty}}{L_{z}} \\
& -\frac{2 T_{\infty} h_{\mathrm{eff}}^{2}(x, y)}{6 k(x)+2 L_{z} h_{\mathrm{eff}}(x, y)} \\
& T(x, y, 0)=T_{\infty}, 0 \leq x \leq L_{x}, 0 \leq y \leq L_{y} \\
& \left.\frac{\partial T}{\partial x}\right|_{x=0}=0,\left.\frac{\partial T}{\partial x}\right|_{x=L_{x}}=0 \\
& \left.\frac{\partial T}{\partial y}\right|_{y=0}=0,\left.\frac{\partial T}{\partial y}\right|_{x=L_{y}}=0
\end{aligned}
$$

Problem (13) is now solved by making use of the Generalized Integral Transform Technique (GITT), in the most general form as 
implemented in the context of the UNIT code [15-17], and now briefly presented. Eq. (13a) may be rewritten as:

$\hat{w} \frac{\partial T(x, y, t)}{\partial t}=\frac{\hat{w}}{w(x)}\left\{\frac{\partial}{\partial x}\left(k(x) \frac{\partial T}{\partial x}\right)+k(x) \frac{\partial^{2} T}{\partial y^{2}}-d(x, y) T+P(x, y, t)\right\}$

$0<x<L_{x}, 0<y<L_{y}, t>0$

or simply:

$\hat{w} \frac{\partial T(x, y, t)}{\partial t}=G(x, y, t, T), 0<x<L_{x}, 0<y<L_{y}, t>0$

$G(x, y, t, T)=\frac{\hat{w}}{w(x)}\left\{\frac{\partial}{\partial x}\left(k(x) \frac{\partial T}{\partial x}\right)+k(x) \frac{\partial^{2} T}{\partial y^{2}}-d(x, y) T+P(x, y, t)\right\}$

Problem (15) with the initial and boundary conditions given by eqs. $(13 d-h)$ may thus be solved by means of the GITT, and the solution may be written as:

$T(x, y, t)=\sum_{i=1}^{N} \tilde{\psi}_{i}(x, y) \bar{T}_{i}(t)$

The transformed potentials are defined with the integral transformation operation given by:

$\bar{T}_{i}(t)=\int_{0}^{L_{x}} \int_{0}^{L_{y}} \hat{w} \tilde{\psi}_{i}(x, y) T(x, y, t) d x d y$

The eigenvalues $\mu_{i}$ and normalized eigenfunctions $\tilde{\psi}_{i}(x, y)$, are obtained from the chosen eigenvalue problem below:

$\hat{k}\left(\frac{\partial^{2} \tilde{\psi}_{i}(x, y)}{\partial x^{2}}+\frac{\partial^{2} \tilde{\psi}_{i}(x, y)}{\partial y^{2}}\right)+\mu_{i}^{2} \hat{w} \tilde{\psi}_{i}(x, y)=0, x \in\left[0, L_{x}\right], y \in\left[0, L_{y}\right]$

$\frac{\partial \tilde{\psi}_{i}(x, y)}{\partial x}=0, x=0, \frac{\partial \tilde{\psi}_{i}(x, y)}{\partial x}=0, x=L_{x}$

$\frac{\partial \tilde{\psi}_{i}(x, y)}{\partial y}=0, y=0, \frac{\partial \tilde{\psi}_{i}(x, y)}{\partial y}=0, y=L_{y}$

where $\hat{k}$ and $\hat{w}$ have been chosen as representative constant values for the thermal conductivity and heat capacity, respectively, so as to allow for the exact analytical solution of problem (17).

The integral transformation is now performed by operating eq. (15a) on with $\int_{0}^{L_{x}} \int_{0}^{L_{y}} \tilde{\psi}_{i}(x, y) \_d x d y$, to yield the following transformed ordinary differential system:

$\frac{d \bar{T}_{i}(t)}{d t}=\bar{g}_{i}\left(t, \bar{T}_{j}\right), t>0, i, j=1,2, \ldots$

with the transformed source terms and initial conditions given by

$\bar{g}_{i}\left(t, \bar{T}_{j}\right)=\int_{0}^{L_{x}} \int_{0}^{L_{y}} G(x, y, t, T) \tilde{\psi}_{i}(x, y) d x d y$

$\bar{f}_{i}=\int_{0}^{L_{x}} \int_{0}^{L_{y}} \hat{w} T_{\infty} \tilde{\psi}_{i}(x, y) d x d y$
The ordinary differential equations system (18) can be numerically solved [18] to provide results for the transformed temperatures, upon truncation to a sufficiently large finite order $N$. Recalling eq. (16a), the temperature field $T(x, y, t)$ is then calculated explicitly in the space variables.

\section{Inverse problem solution}

In the Bayesian approach, inference is drawn by constructing the joint probability distribution of all unobserved quantities based on all that is known about them. This knowledge incorporates previous information about the phenomena under study and is also based on values of observed quantities when they are available. This approach is based on Bayes' theorem, which can be written as [19-21]

$p(\mathbf{P} \mid \mathbf{Y})=\frac{p(\mathbf{Y} \mid \mathbf{P}) p(\mathbf{P})}{p(\mathbf{Y})}$

In summary, solving an inverse problem within the Bayesian framework may be broken into three subtasks: (i) Based on all information available for the unknown $\mathbf{P}$, find a prior probability density $p(\mathbf{P})$ that reflects judiciously this prior information; (ii) Find the likelihood function $p(\mathbf{Y} \mid \mathbf{P})$ that describes the interrelation between the observations and the unknowns; (iii) Develop methods to explore the posterior probability density $p(\mathbf{P} \mid \mathbf{Y})$.

When it is not possible to analytically obtain the corresponding posterior distributions, one needs to use a method based on simulation. The inference based on simulation techniques uses samples from the posteriori $p(\mathbf{P} \mid \mathbf{Y})$ to extract information about them. Several sampling strategies are proposed in the literature, including the Monte Carlo method via Markov Chain (MCMC), adopted in this work.

The most commonly used MCMC sampling algorithm is the Metropolis-Hastings, here employed [19-21]. The MetropolisHastings algorithm uses the same idea of the rejection methods, i.e. a value is generated from an auxiliary distribution and accepted with a given probability. This correction mechanism ensures the convergence of the chain to the equilibrium distribution.

The unknown quantities in this work, the variable thermal properties and the effective heat transfer coefficient, were expressed as eigenfunction expansions, which significantly reduces the number of parameters. The truncation orders and the choices of filtering functions in the proposed expansions, eqs. $9(a-f)$, govern the number of parameters to be estimated. Thus, the total number of parameters $N_{P}$ is given by the sum of parameters in each expansion, including the number of parameters in each filter, and the number of parameters in the heat flux expression, eventually also to be estimated.

Another important aspect of the present study is the solution of the inverse problem in the transformed field, from the integral transformation of the experimental temperature data, thus compressing the experimental measurements in the space variables into a few transformed temperature fields. Once the experimental temperature spatially distributed readings have been obtained, one proceeds to the integral transformation of the temperature field at each time through the integral transform pair below:

Transform : $\bar{T}_{\exp , i}(t)=\int_{0}^{L x} w(x) \tilde{\psi}_{i}(x)\left[T_{\exp }(x, t)-T_{\infty}\right] d x$

Inverse : $T_{\exp }(x, t)=T_{\infty}+\sum_{i=0}^{N} \tilde{\psi}_{i}(x) \bar{T}_{\exp , i}(t)$ 


\section{Results and discussion}

The aim is to first employ the one-dimensional experiment and its corresponding inverse analysis to estimate the space variable thermophysical properties of the nanocomposite slab. Then, the estimated quantities are employed in simulating the multidimensional problem, which is representative of an application, allowing for critical comparisons between the simulated and experimental results.

The experimental conditions here encountered are expected to fit into the problem formulation given by eq. (5), which was adopted in the direct and inverse problem solutions. The time variation of the applied heat flux, which accounts for the thermal capacity of the resistance itself and of the thermal paste, besides the thermal contact resistance, has been parameterized for the estimation. Therefore, the applied heat flux is considered to be given here by:

$$
\begin{aligned}
& q(x, t)=q_{w}(x) f(t), f(t)=c-a e^{-b t}, \\
& q_{w}(x)= \begin{cases}q_{1}, & 0<x<x_{C} \\
q_{2}, & x_{C}<x<L_{x}\end{cases}
\end{aligned}
$$

where the positions $x=0$ and $x=x_{C}=4 \mathrm{~cm}$ correspond to where the electric resistance begins and ends, respectively. Therefore, the unheated portion of the plate corresponds to a heat flux $q_{2}=0$. Since the dissipated power in the resistance is accurately measured, eq. (5a) is thus divided by $q_{1}$, and then the estimated parameters can be expressed by multiplying each one with the measured heat flux value, $q_{1}$ and its associated uncertainty.

The effective heat transfer coefficient has a more involved behavior and has been here expanded in eigenfunctions according to eq. $9(\mathrm{e}, \mathrm{f})$. In light of the nature of the applied heating, a filter was considered in the form of a step function that assumes two different characteristic values at the heated and unheated plate portions:

$h_{\mathrm{eff}, f}(x)=\left\{\begin{array}{cc}h_{x 0} & 0<x<x_{C} \\ h_{x L} & x_{C}<x<L_{x}\end{array}\right.$

The initial estimates were then obtained from correlations for natural convection over vertical plates and linearization of the radiative heat flux. As previously discussed, the expansion truncation orders govern the number of parameters involved in the estimation procedure. Here, the total number of parameters refers to the filters and expansions for the thermal conductivity, thermal capacity, and effective heat transfer coefficient. Also, the parameters $a, b$ and $c$ in eq. $(21 \mathrm{a}-\mathrm{c})$, that control the time variation of the applied heat flux, are included:

$$
\begin{aligned}
\mathbf{P}=[ & \left(k_{x 0}, k_{x L}, \bar{k}_{1}, \bar{k}_{2}, \ldots, \bar{k}_{N_{k}}\right),\left(w_{x 0}, w_{x L}, \bar{w}_{1}, \bar{w}_{2}, \ldots, \bar{w}_{N_{w}}\right), \\
& \left.\left(d_{x 0}, d_{x L}, \bar{d}_{1}, \bar{d}_{2}, \ldots, \bar{d}_{N_{d}}\right), a, b, c\right]
\end{aligned}
$$

Based on the observation of fairly sharp interface that exists between the two materials that compose the plate, we consider a filter function for the thermophysical properties that approximates a step transition between the end values at $x=0$ and $x=L_{\chi}$, in the form [25]:

$$
k_{f}(x)=k_{x 0}+\left(k_{x L}-k_{x 0}\right) \delta(x), w_{f}(x)=w_{x 0}+\left(w_{x L}-w_{x 0}\right) \delta(x)
$$

where

$\delta(x)=\frac{1}{1+e^{-\gamma\left(-x+x_{t}\right)}}$

In eq. (24c), $\gamma$ is a parameter that controls the transition sharpness and $x_{t}$ is the transition point. Both are considered as fixed parameters, not to be estimated with the inverse analysis, the values of which are taken as $\gamma=1500 \mathrm{~m}^{-1}$ and $x_{t}=0.02 \mathrm{~m}$ [25].

For the parameters $k_{x 0}, k_{x L}, w_{x 0}$ and $w_{x L}$ we have adopted normal priors, with $15 \%$ standard deviation, centered in literature mean values for the polyester resin [26] and we have used the Lewis-Nielsen formula [27] for the region filled with alumina nanoparticles. The Lewis-Nielsen formula takes into account the effect of the shape of the particles and the orientation or type of packing for a two-phase system:

$$
k_{c}=k_{m}\left[\frac{1+A B \varphi}{1-B \varphi \psi}\right]
$$

$$
B=\frac{\left(k_{d} / k_{m}\right)-1}{\left(k_{d} / k_{m}\right)+A}
$$

$$
\psi=1+\left(\frac{1-\varphi_{s}}{\varphi_{s}^{2}}\right) \varphi
$$

\begin{tabular}{|c|c|c|}
\hline Parameter & Estimated values (classical lumped) [26] & Estimated values (improved lumped) \\
\hline$a$ & $0.19[0.188,0.192]$ & $0.189[0.187,0.193]$ \\
\hline$b$ & $0.003319[0.003314,0.003324]$ & $0.004519[0.00451,0.00453]$ \\
\hline$c$ & $0.660[0.658,0.661]$ & $0.657[0.654,0.660]$ \\
\hline
\end{tabular}

Table 1a

Estimated parameters with 99\% confidence intervals for the thermophysical properties.

\begin{tabular}{lllll}
\hline Property & Material & Estimated (classical lumped) [25] & Estimated (improved lumped) & ${ }^{\mathrm{a}}$ Reference values \\
\hline$k$ & Polyester & 0.1617 & 0.1596 & $0.15-0.17$ \\
& Nanocomposite & 0.2042 & 0.2037 & $0.18-0.21$ \\
$w$ & Polyester & $1.59 \times 10^{6}$ & $1.529 \times 10^{6}$ & $(1.22-1.76) \times 10^{6}$ \\
& Nanocomposite & $1.76 \times 10^{6}$ & $1.70 \times 10^{6}$ & $(1.36-1.91) \times 10^{6}$ \\
\hline
\end{tabular}

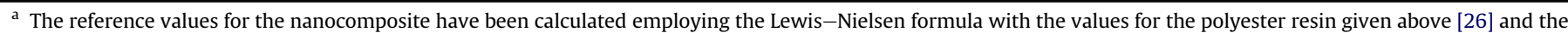
following values for the aluminum oxide [28]: $k=32-46 \mathrm{~W} / \mathrm{m}^{\circ} \mathrm{C}$ and $w=(3-3.7) \times 10^{6} \mathrm{~J} / \mathrm{m}^{3 \circ} \mathrm{C}$.

Table 1b

Estimated parameters with $99 \%$ confidence intervals for the applied heat flux. 

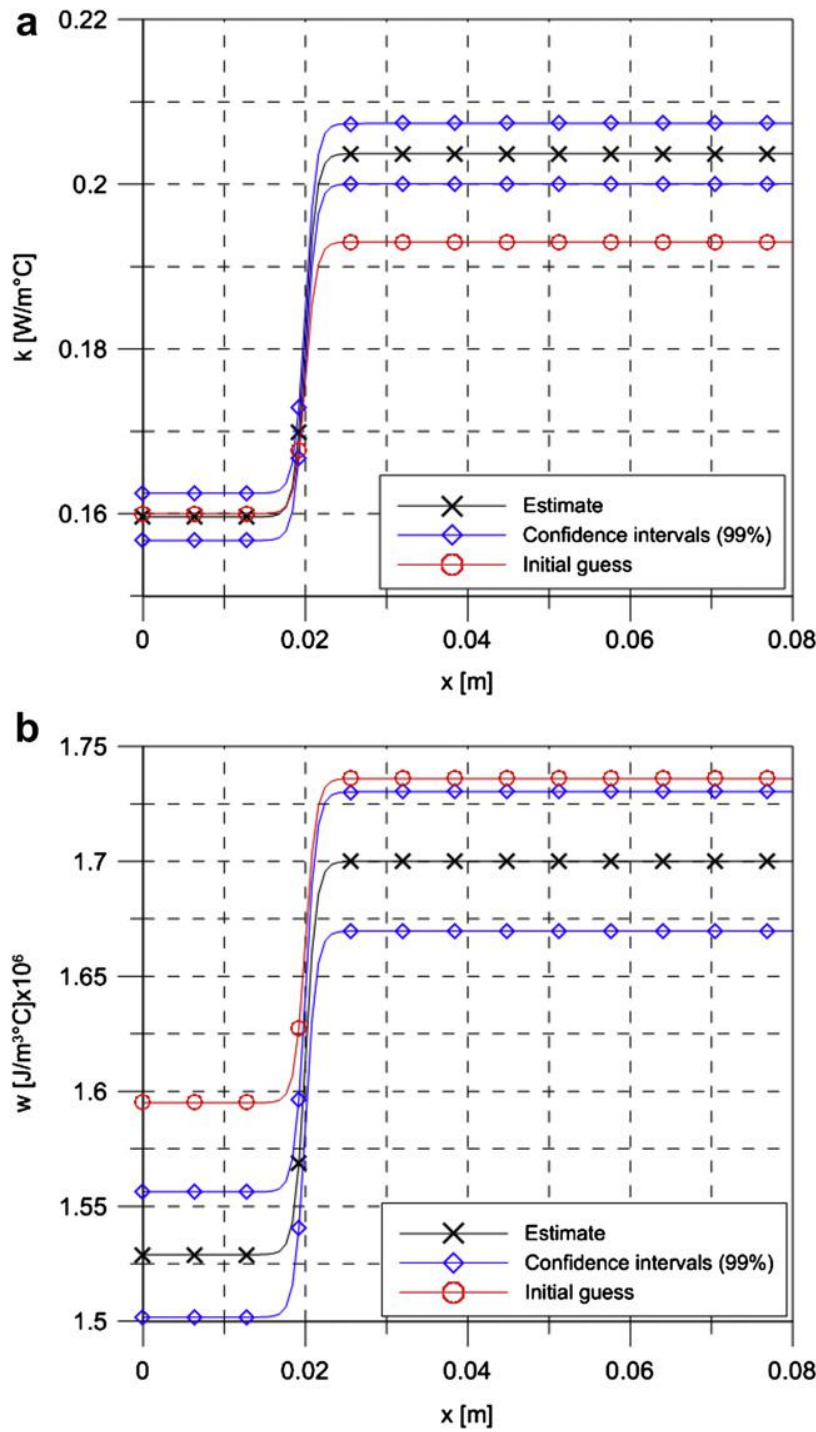

Fig. 6. (a) Estimated thermal conductivity. (b) Estimated heat capacity.

where $\phi$ is the volumetric concentration of filler, $k_{c}$ is the nanocomposite calculated effective thermal conductivity, $k_{d}$ is the thermal conductivity of the dispersed particles used as filler, in this instance the aluminum oxide, and $k_{m}$ is the polymeric matrix thermal conductivity, which in our case is the polyester resin. $A$ and $\phi_{s}$ are given for several geometric shapes and orientation [27]. For spheres with random packing we have $A=1.5$ and $\phi_{\mathrm{s}}=0.637$.

Also, normal priors were employed for the two values of the parameters in the filter of the effective heat transfer coefficients, $h_{x 0}$
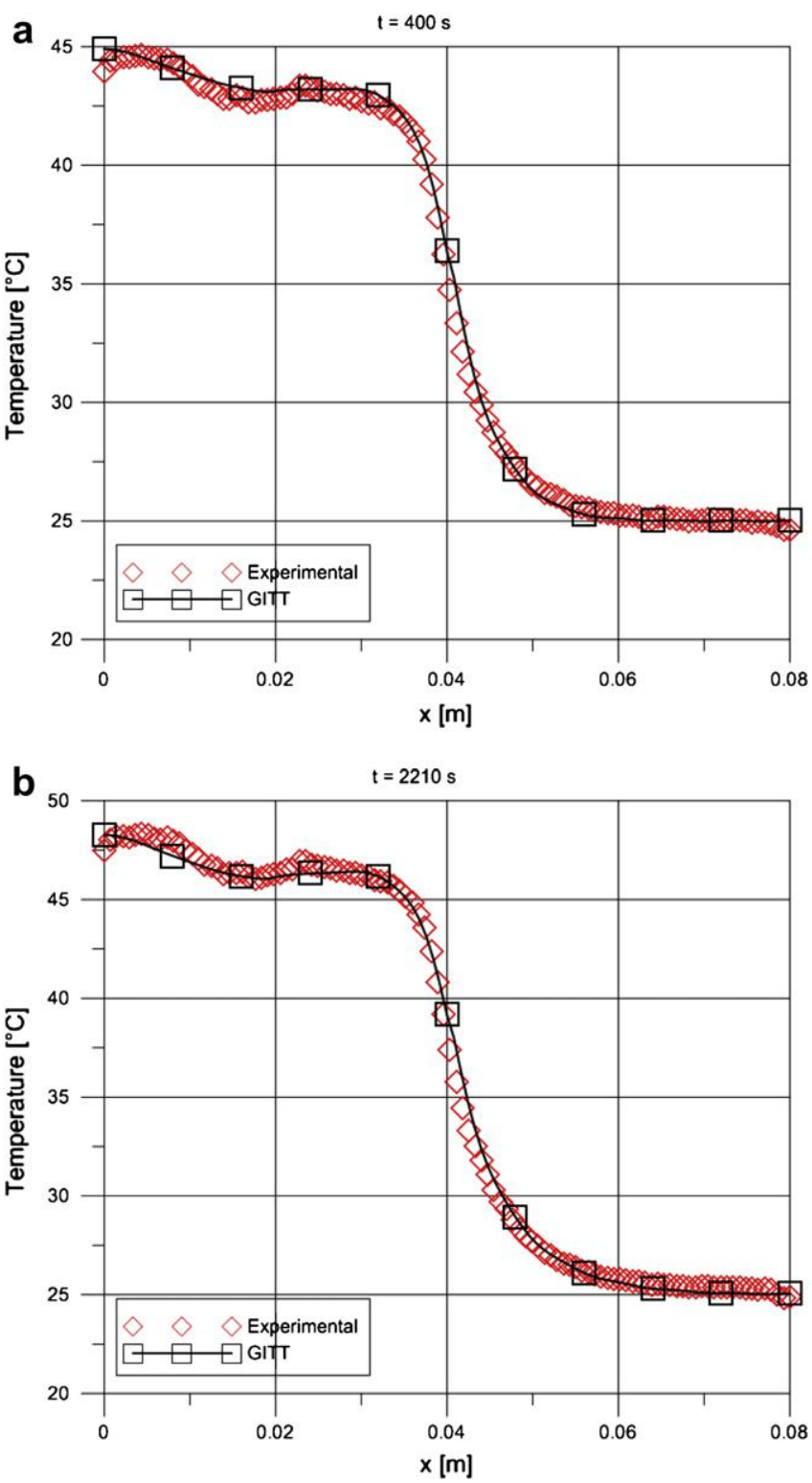

Fig. 7. (a) Vertical spatial distribution of temperatures at $t=400 \mathrm{~s}$. (b) Vertical spatial distribution of temperatures at $t=2210 \mathrm{~s}$

and $h_{x L}$, provided by available correlations of natural convection and linearized radiation. For the remaining parameters, $\left(\bar{k}_{1}, \bar{k}_{2}, \bar{k}_{3}, \bar{w}_{1}, \bar{w}_{2}, \bar{w}_{3}, \bar{h}_{1}, \bar{h}_{2}, \bar{h}_{3}, a, b, c\right)$, non-informative priors have been adopted.

Tables $1 \mathrm{a}$ and $1 \mathrm{~b}$ present the mean values of the estimated parameters for the thermophysical properties and the applied heat

Table 2

Convergence behavior of the temperature field for the solution obtained using the integral transform method and the improved lumped approach, eq. (4c).

\begin{tabular}{|c|c|c|c|c|c|c|}
\hline \multirow[t]{3}{*}{ Truncation order } & \multicolumn{3}{|c|}{$T^{*}\left(x, L_{z}, t\right)\left[{ }^{\circ} \mathrm{C}\right]$ eq. $(4 \mathrm{c})$} & \multicolumn{3}{|c|}{$T^{*}\left(x, L_{z}, t\right)\left[{ }^{\circ} \mathrm{C}\right]$ eq. $(4 \mathrm{c})$} \\
\hline & \multicolumn{3}{|l|}{$t=200 \mathrm{~s}$} & \multicolumn{3}{|l|}{$t=400 \mathrm{~s}$} \\
\hline & $x=0.2 \mathrm{~cm}$ & $x=0.4 \mathrm{~cm}$ & $x=0.6 \mathrm{~cm}$ & $x=0.2 \mathrm{~cm}$ & $x=0.4 \mathrm{~cm}$ & $x=0.6 \mathrm{~cm}$ \\
\hline$N=65$ & 38.2421 & 32.8143 & 25.0284 & 43.1344 & 36.3730 & 25.1145 \\
\hline$N=60$ & 38.2473 & 32.8097 & 25.0256 & 43.1315 & 36.3694 & 25.1120 \\
\hline$N=55$ & 38.2572 & 32.7732 & 24.9912 & 43.1456 & 36.2732 & 25.0550 \\
\hline$N=50$ & 38.2613 & 32.5809 & 25.0788 & 43.1552 & 36.1114 & 25.1710 \\
\hline$N=45$ & 39.2506 & 32.2742 & 23.8192 & 44.3336 & 35.7342 & 23.6404 \\
\hline
\end{tabular}


flux, respectively. For the sake of comparison, the estimated parameters obtained as the solution of this inverse problem when considering the classical lumped formulation for the solution of the direct problem [25] are also presented. One may observe that the estimates for the heat capacity and for the parameter $b$ of the applied heat flux presented the most significant deviations from the previous analysis [25], when using the improved lumped approach. Also, Table 1a brings the reference values for the thermophysical properties of the polyester resin [26] and the alumina-polyester nanocomposite as calculated from the Lewis-Nielsen formula [27] and Figs. 6a,b present, respectively, the estimated spatial variations for the thermal conductivity and heat capacity, with their 99\% confidence intervals, as well as the initial guess employed. We must stress that we have employed three transformed fields in the expansion of each property in eqs. (9a-d), $N_{\mathrm{k}}=N_{\mathrm{w}}=3$, and their estimated values in the inverse problem
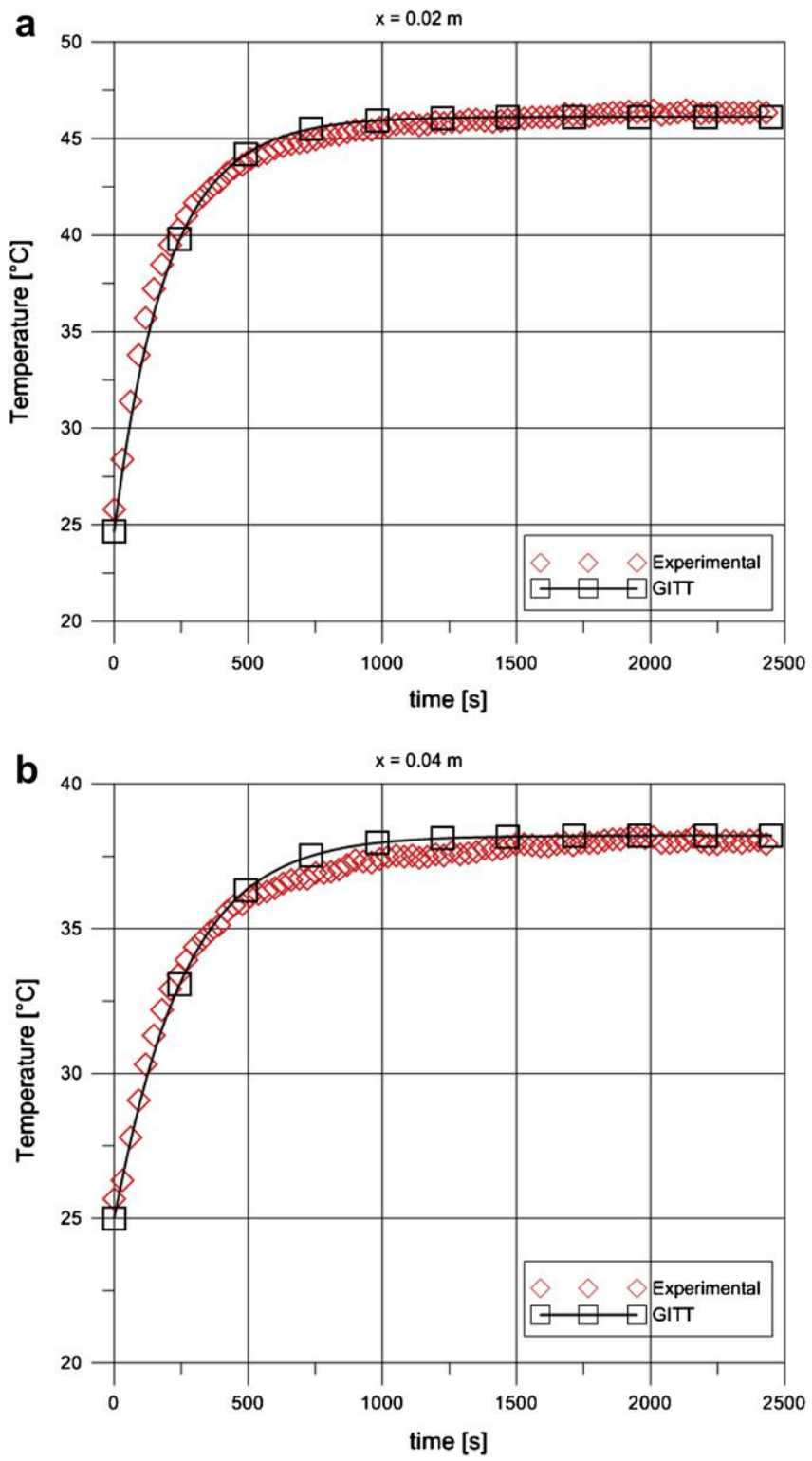

Fig. 8. (a) Time evolution of the temperature at $x=2 \mathrm{~cm}$ (b) Time evolution of the temperature at $x=4 \mathrm{~cm}$. solution have been very small $\left(\bar{k}_{k}, \bar{w}_{k}<10^{-7}, k=1,2,3\right)$, confirming that the step filter employed already describes fairly well the properties functional form [25]. The integral transform method has been used for the solution of problem (5) considering the estimated values herein obtained. Table 2 illustrates the convergence behavior of the temperature values at selected positions and times, for increasing truncation orders from bottom to top. The results are apparently fully converged to four digits for $N=65$ in all selected positions. Figs. 7 bring some curves for the temperature vertical spatial distribution at different time values and Figs. 8 depict the time evolution of the temperature at two different positions, and an excellent agreement between the simulated results and the experimental data is observed throughout. Fig. 9 presents the residuals between simulated and measured temperatures at three positions, $x=0.02,0.04$ and $0.06 \mathrm{~m}$. Although presenting some correlation, the residuals are small.

Figs. 10 brings the comparison between the temperature profiles at the plate surface along its length obtained with the classical and the improved lumped approaches using the same input parameters, at three different times, $t=200,400$ and $2210 \mathrm{~s}$, respectively. The difference between the solutions is better assessed with the aid of Fig. 11, which shows the temperature deviations between the classical and improved lumped solutions using the same input parameters, for these three different times. It can be noticed that the solution obtained with the classical lumped approach yields slightly higher temperatures, with deviations of up to $0.5^{\circ} \mathrm{C}$, during the transient. At steady state, both curves are essentially coincident on the graphic scale. In fact, this result confirms the analysis of the inverse problem solution, where we have noticed that the heat capacity and the parameter $b$, both parameters with high influence during the transient period, had the largest deviations when estimated with the classical and the improved lumped approaches.

Now, we look at the case in which the smaller $14 \times 14 \mathrm{~mm}$ electrical resistance is used, simulating an electronic chip installed on top of the heterogeneous nanocomposite substrate, which in this situation works as a heat spreader. For the mathematical formulation of this problem we cannot neglect the heat diffusion along the plate's width and thus an improved lumped-differential

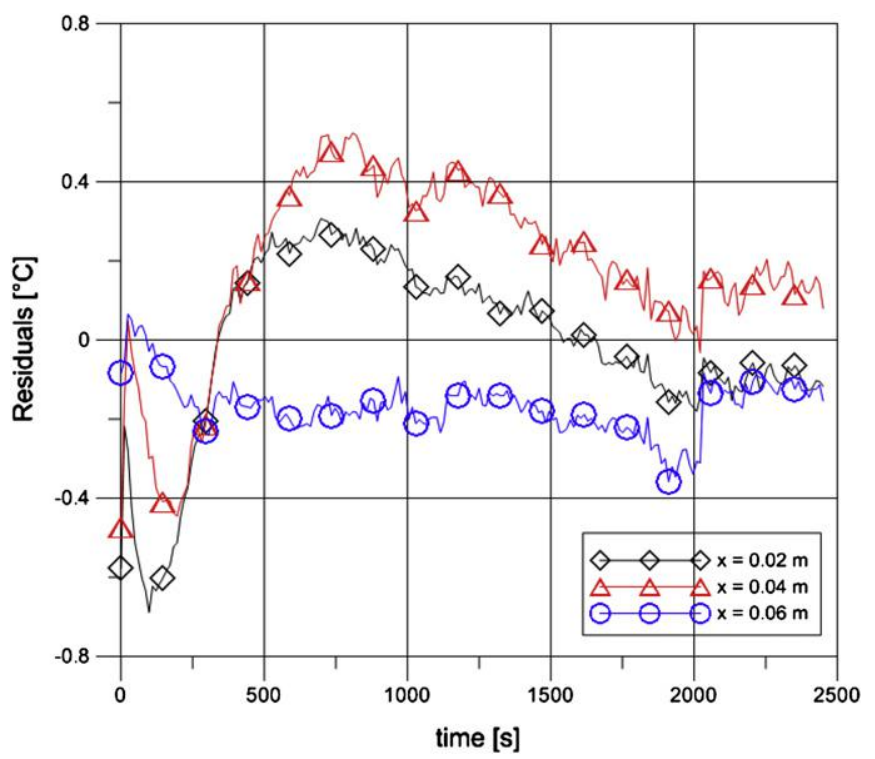

Fig. 9. Residuals $\left[{ }^{\circ} \mathrm{C}\right]$ between calculated and experimental temperatures at three different positions. 
a

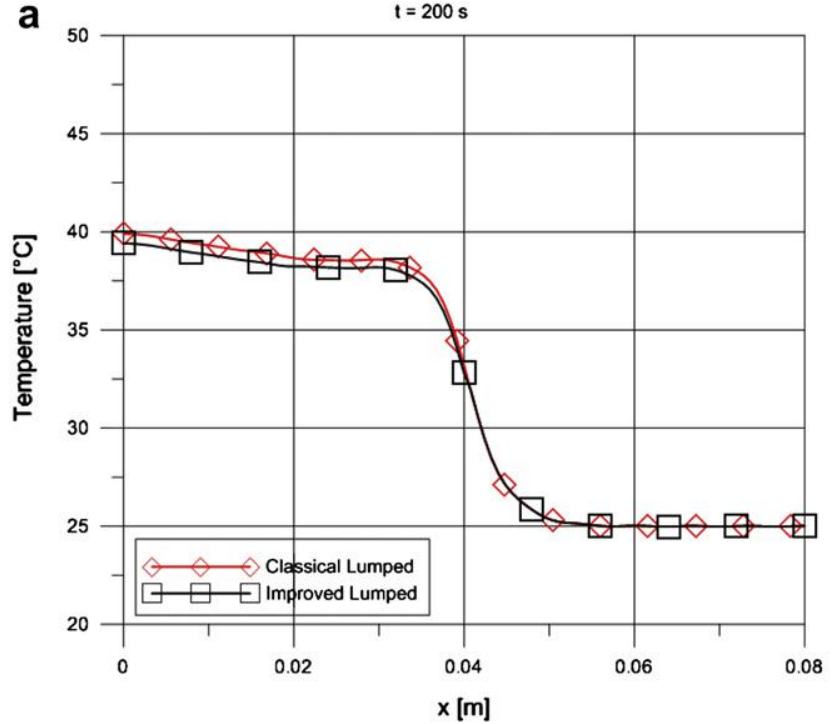

b

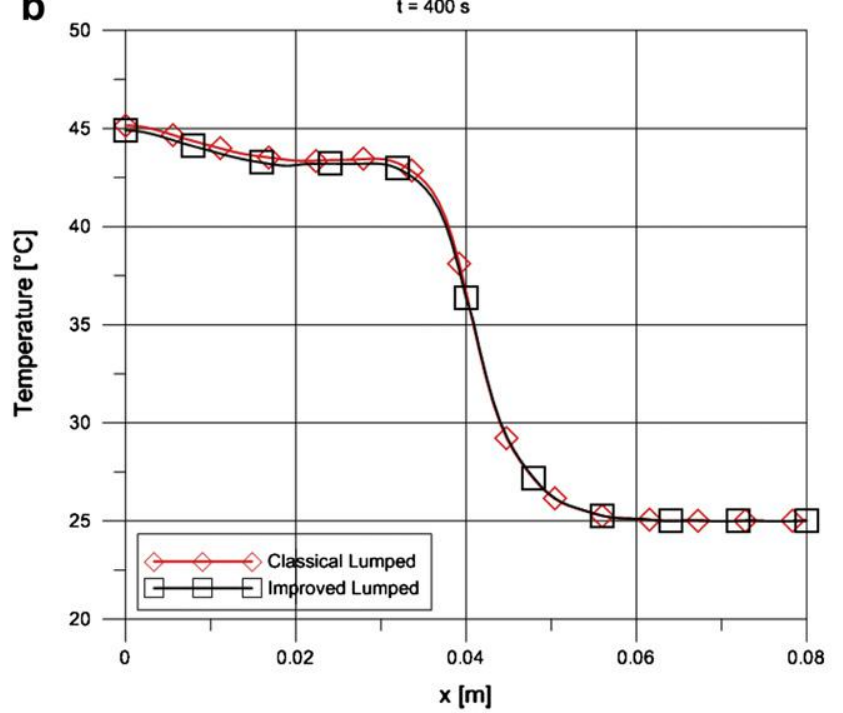

C

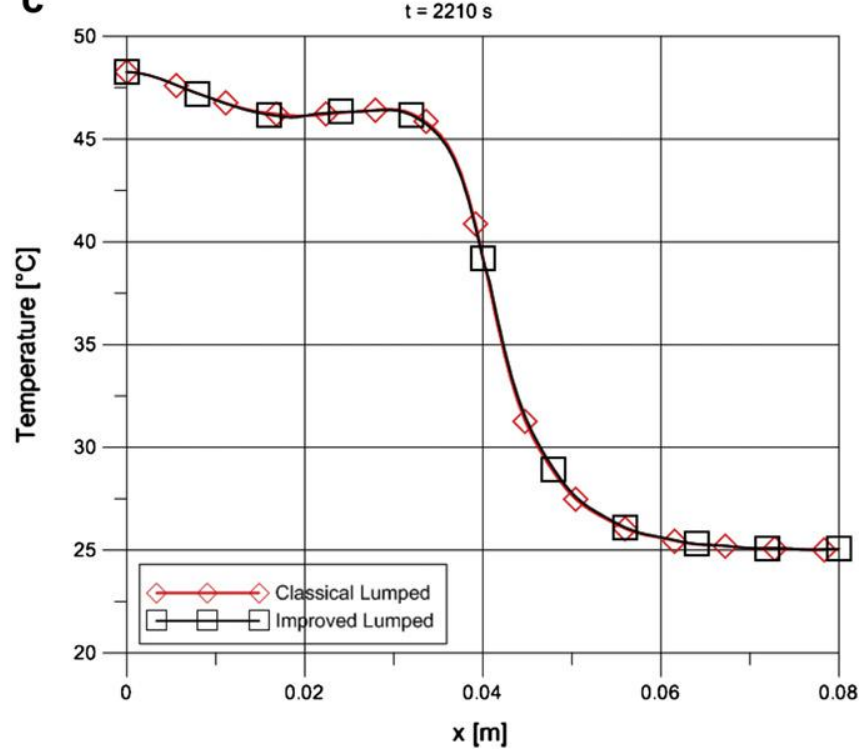

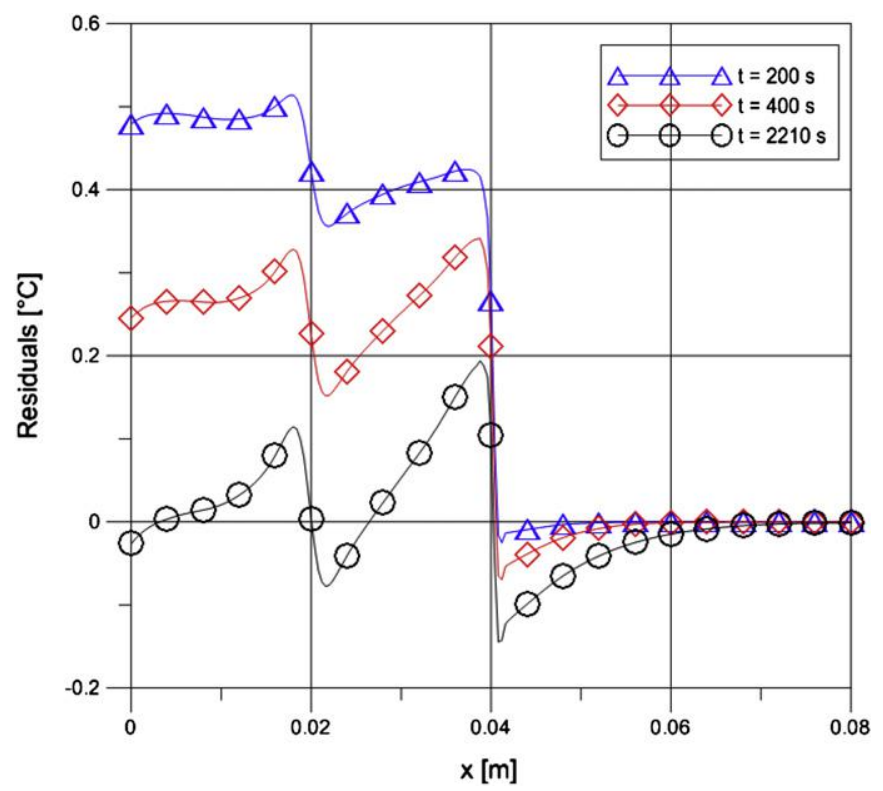

Fig. 11. Deviations $\left[{ }^{\circ} \mathrm{C}\right]$ between the solutions obtained with the classical and improved lumped approaches using the same input parameters for three different times: $t=200,400$ and $2210 \mathrm{~s}$.

two-dimensional model is necessary, which corresponds to the problem formulation in eqs. (13).

For this multidimensional problem solution the Generalized Integral Transform Technique (GITT) was employed as previously described, constructed as a dedicated code in the Mathematica platform [18], using the typical steps adopted by the UNIT algorithm in order to illustrate the flexibility of this solution path [15-17]. The space-dependent thermophysical properties, $k(x)$ and $w(x)$, have been adopted as estimated by the previous inverse analysis procedure. For the heat transfer coefficient we have used natural convection correlations and, based on the estimated value for parameter $c$ in the inverse analysis procedure, we have considered that $65.7 \%$ of the heat flux generated by the electrical resistance is applied on the nanocomposite plate. Again, the improved lumped formulation has been used in order to yield enhanced approximations of the temperature distribution at the plate's surface, $z=L_{z}$.

Table 3 illustrates the convergence behavior of the temperature profile at chosen positions and time values for the two-dimensional situation, for increasing truncation orders from bottom to top. The results are apparently converged to practically four digits for $N<200$ in all selected positions.

Figs. 12 illustrate the temperature distributions along, respectively, the length $(y=2 \mathrm{~cm})$ and the width $(x=0.7 \mathrm{~cm})$ of the plate, both for $t=1000 \mathrm{~s}$, where a reasonably good agreement between the simulated and experimental data can be observed, once the estimated heat flux partition $(c=0.66)$ is employed in the modeling of the applied heat flux to the nanocomposite substrate, thus accounting for heat losses through the insulation.

Fig. 13 shows the time evolution of the temperature measured at the surface of the plate at the center of the region where the electrical resistance is placed. One may observe that a better

Fig. 10. Comparison between the temperature profile at the surface of the plate obtained with the classical and the improved lumped formulations at: (a) $t=200 \mathrm{~s}$. (b) $\mathrm{t}=400 \mathrm{~s}$. (c) $\mathrm{t}=2210 \mathrm{~s}$. 
Table 3

Convergence behavior of the temperature profile for the solution of the two-dimensional problem.

\begin{tabular}{|c|c|c|c|c|c|c|}
\hline \multirow[t]{4}{*}{ Truncation order } & \multicolumn{3}{|l|}{$T_{s}(x, y, t)\left[{ }^{\circ} \mathrm{C}\right]$} & \multicolumn{3}{|l|}{$T_{s}(x, y, t)\left[{ }^{\circ} \mathrm{C}\right]$} \\
\hline & \multicolumn{3}{|l|}{$t=100 \mathrm{~s}$} & \multicolumn{3}{|l|}{$t=200 \mathrm{~s}$} \\
\hline & $x=0.7 \mathrm{~cm}$ & $x=1.4 \mathrm{~cm}$ & $x=2 \mathrm{~cm}$ & $x=0.7 \mathrm{~cm}$ & $x=1.4 \mathrm{~cm}$ & $x=2 \mathrm{~cm}$ \\
\hline & $y=2 \mathrm{~cm}$ & $y=2 \mathrm{~cm}$ & $y=2 \mathrm{~cm}$ & $y=2 \mathrm{~cm}$ & $y=2 \mathrm{~cm}$ & $y=2 \mathrm{~cm}$ \\
\hline$N=200$ & 55.4965 & 41.2204 & 27.7384 & 62.3461 & 45.1843 & 29.0914 \\
\hline$N=190$ & 55.4998 & 41.2093 & 27.7314 & 62.3510 & 45.1788 & 29.0854 \\
\hline$N=180$ & 55.5069 & 41.2309 & 27.6870 & 62.3625 & 45.1903 & 29.0389 \\
\hline$N=170$ & 55.5346 & 41.2378 & 27.5858 & 62.3861 & 45.1969 & 29.0021 \\
\hline$N=160$ & 55.8803 & 41.4502 & 27.6988 & 62.7433 & 45.4083 & 29.0129 \\
\hline
\end{tabular}

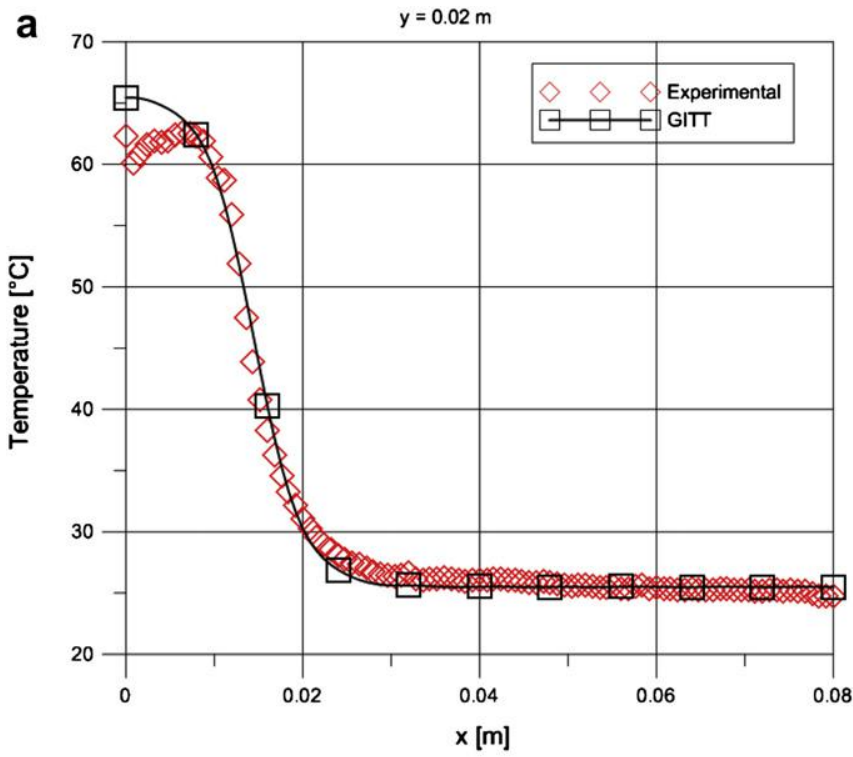

agreement between simulated and experimental data is achieved after some elapsed time. The less adherent behavior observed in the beginning of the heating process may be explained by the thermal capacity of the smaller electrical resistance installation, not available from the inverse problem analysis, and which was therefore neglected in the two-dimensional model solution.

Figs. 14a,b present eight isotherms at steady state for the experimental data and the simulated results, respectively, showing an overall good agreement for the entire substrate domain. Deviations between experimental and theoretical results are within $1.5{ }^{\circ} \mathrm{C}$ except within the very edge of the electrical resistance, where the model does not incorporate a heat transfer coefficient to account for heat losses and the deviation can reach $4{ }^{\circ} \mathrm{C}$. This good agreement between the experimental and simulated curves for the two-dimensional case confirms that reliable thermophysical properties identification in heterogeneous materials is critical for adequate simulation in the realm of applications. Further improvement in this situation would require the estimation of the space variable heat transfer coefficient and applied heat flux directly at the two-dimensional experimental configuration, or alternatively, solving the more cumbersome conjugated conduction-convection-radiation problem [29,30].
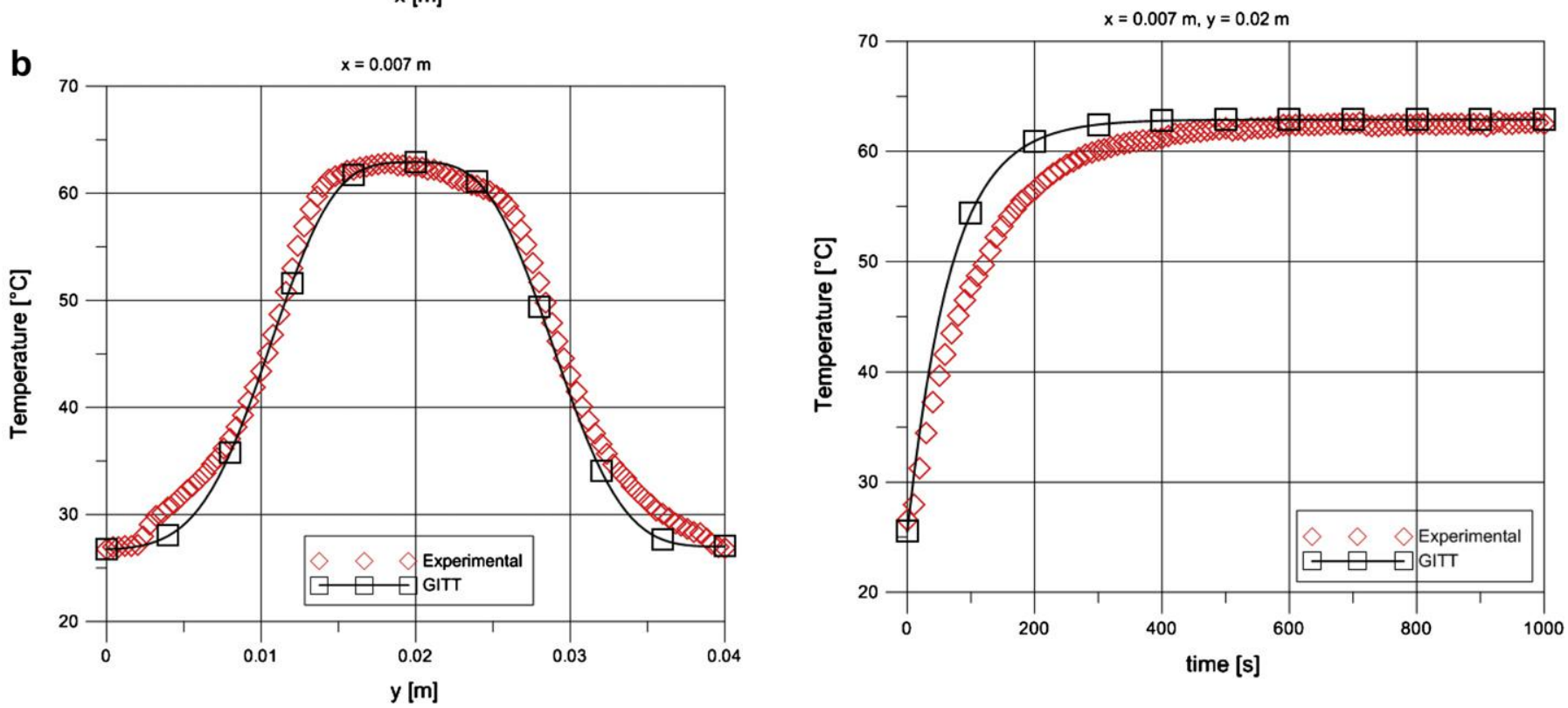

Fig. 12. Temperature distribution along the plate's (a) length and (b) width, for the case used to simulate an electronic device installed on the nanocomposite substrate as a heat spreader.

Fig. 13. Time evolution of the temperature measured at the surface of the plate at the center of the region where the electrical resistance is placed for the case used to simulate an electronic device installed on the nanocomposite substrate as a heat spreader. 

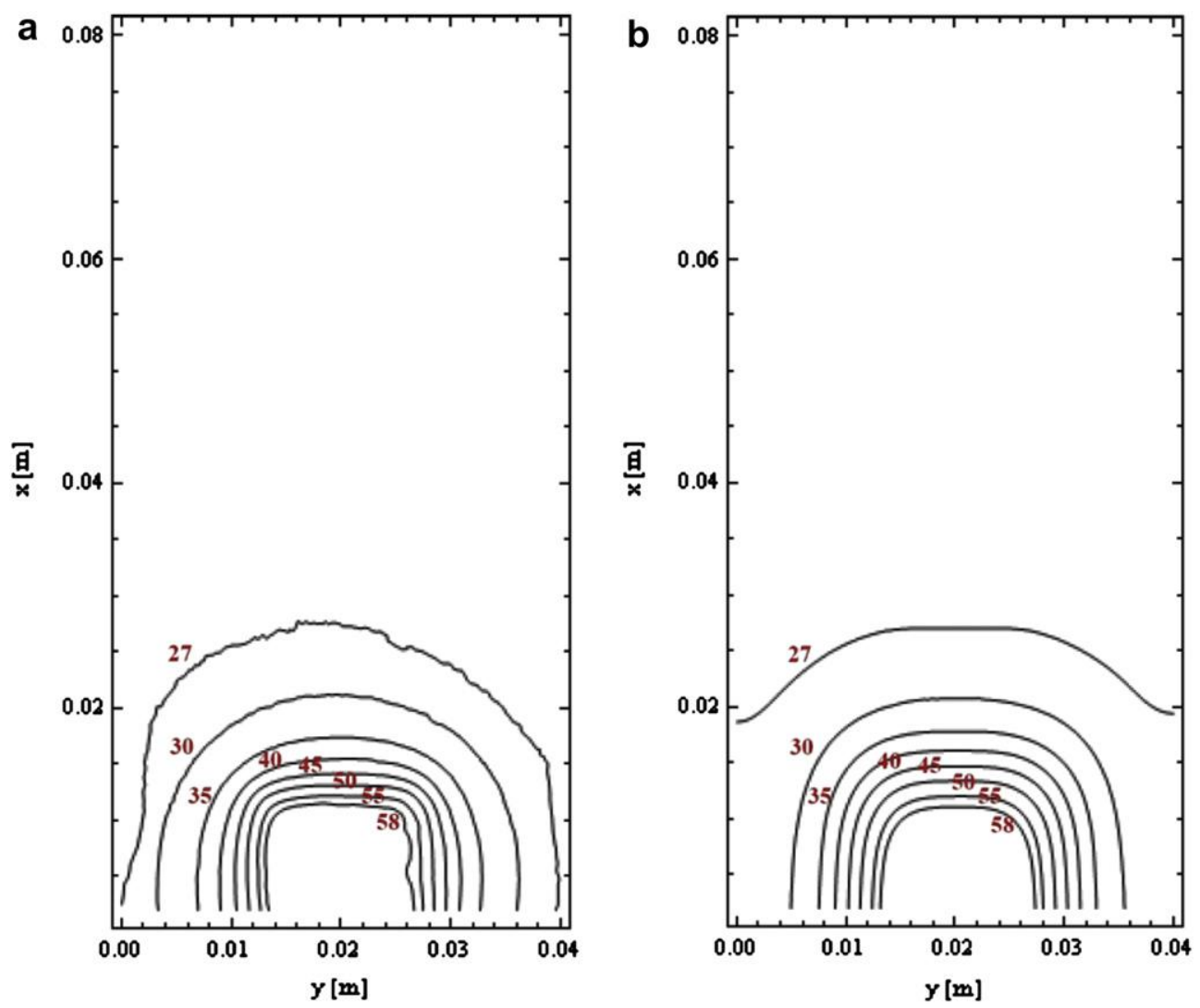

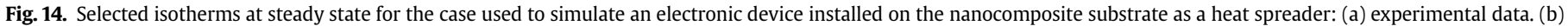
simulated results.

\section{Conclusions}

The present work is concerned with the estimation of space variable thermophysical properties in heterogeneous nanocomposite plates, and the utilization of this information in the thermal analysis of heat spreaders made of the same materials. Improved lumped-differential formulations are developed for both the problem that corresponds to the properties identification experimental setup, and the multidimensional heat spreader situation. The aim is to achieve simpler formulations by averaging the original problems in the plate thickness, but providing more accurate relations between the surface and averaged temperatures, in comparison to the classical lumped system analysis. Then, the lumped-differential formulations for the average temperatures are accurately solved by integral transforms, and the improved expressions are employed to more accurately estimate the surface temperatures, which feed the inverse problem analysis and the comparisons with the infrared thermography experimental results.

A previously developed combination of integral transforms, Bayesian inference and infrared thermography provides the necessary tools for both the space variable properties identification effort and the verification of the multidimensional formulation, with overall good agreement.

\section{Acknowledgments}

The authors would like to acknowledge the partial financial support provided by CNPq, CAPES and FAPERJ, all of them sponsoring agencies in Brazil.

\section{References}

[1] F. Danes, B. Garnier, T. Dupuis, Predicting, measuring and tailoring the transverse thermal conductivity of composites from polymer matrix and metal filler, Int. J. Thermophysics 24 (2003) 771-784.

[2] D. Kumlutas, I.H. Tavman, A numerical and experimental study on thermal conductivity of particle filled polymer composites, J. Thermoplastic Composite Mater. 19 (2006) 441-455.

[3] I. Philippi, J.C. Batsale, D. Maillet, A. Degiovanni, Measurement of thermal diffusivity trough processing of infrared images, Rev. Sci. Instrum. 66 (1995) 1182-1192.

[4] C. Pradère, M. Joanicot, J.C. Batsale, J. Toutain, C. Gourdon, Processing of temperature field in chemical microreactors with infrared thermography, QIRT J. 3 (2006) 17-135.

[5] O. Fudym, Velocity and Heat Transfer Parameters Mapping: Thermal Quadrupoles and Infrared Image Processing, 11th Brazilian Congress of Thermal Sciences and Engineering - ENCIT, Curitiba, Brazil, December 5-8, 2006.

[6] O. Fudym, H.R.B. Orlande, M. Bamford, J.C. Batsale, Bayesian approach for thermal diffusivity mapping from infrared images processing with spatially random heat pulse heating. J. Physics. Conf. Ser. (Online) 135 (2008) 12-42.

[7] M.N. Ozisik, H.R.B. Orlande, Inverse Heat Transfer: Fundamentals and Application, Taylor and Francis, New York, 2000.

[8] R.M. Cotta, Integral Transforms in Computational Heat and Fluid Flow, CRC Press, 1993.

[9] R.M. Cotta, M.D. Mikhailov, Heat Conduction: Lumped Analysis, Integral Transforms, Symbolic Computation, Wiley-Interscience, 1997.

[10] R.M. Cotta, M.D. Mikhailov, Hybrid methods and symbolic computations, in: W.J. Minkowycz, E.M. Sparrow, J.Y. Murthy (Eds.), Handbook of Numerical Heat Transfer, second ed. John Wiley, New York, 2006.

[11] C.P. Naveira-Cotta, R.M. Cotta, H.R.B. Orlande, O. Fudym, Eigenfunction expansions for transient diffusion in heterogeneous media, Int. J. Heat Mass Transfer 52 (2009) 5029-5039.

[12] C.P. Naveira-Cotta, H.R.B. Orlande, R.M. Cotta, Integral transforms and Bayesian inference in the identification of variable thermal conductivity in two-phase dispersed systems, Num. Heat Transfer. Part B. Fundam. 57 (3) (2010) 173-203.

[13] C.P. Naveira-Cotta, H.R.B. Orlande, R.M. Cotta, J.S. Nunes, Integral Transforms, Bayesian Inference, and Infrared Thermography in the Simultaneous Identification of Variable Thermal Conductivity and Diffusivity in Heterogeneous Media, 14th Int. Heat Transfer Conf., Washington DC, USA, August, 2010. 
[14] C.P. Naveira-Cotta, R.M. Cotta, H.R.B. Orlande, Inverse analysis with integral transformed temperature fields for identification of thermophysical properties functions in heterogeneous media, Int. J. Heat Mass Transfer 54 (7-8) (2011) 1506-1519.

[15] D.C. Knupp, C.P. Naveira-Cotta, J.V.C. Ayres, H.R.B. Orlande, R.M. Cotta Experimental-theoretical analysis of a transient heat conduction setup via infrared thermography and unified integral transforms, Int. Rev. Chem. Eng. 2 (2010) 736-747.

[16] L.A. Sphaier, R.M. Cotta, C.P. Naveira-Cotta, J.N.N. Quaresma, The UNIT algorithm for solving one-dimensional convection-diffusion problems via integral transforms, Int. Comm. Heat and Mass Transfer 38 (5) (2011) 565-571.

[17] R.M. Cotta, J.N.N. Quaresma, L.A. Sphaier, C.P. Naveira-Cotta, Unified Integral Transform Approach in the Hybrid Solution of Multidimensional Nonlinear Convection-Diffusion Problems, 14th Int. Heat Transfer Conf., Washington DC, USA, August, 2010

[18] S. Wolfram, The Mathematica Book version 5.2, Cambridge-Wolfram Media, 2005.

[19] J. Kaipio, E. Somersalo, Statistical and Computational Inverse Problems, Springer, New York, 2005.

[20] H.S. Migon, D. Gamerman, Statistical Inference: An Integrated Approach, Arnold/Oxford, London/New York, 1999.

[21] D. Gamerman, H.F. Lopes, Markov Chain Monte Carlo: Stochastic Simulation for Bayesian Inference, second ed. Chapman \& Hall/CRC, Boca Raton, FL, 2006.
[22] J.B. Aparecido, R.M. Cotta, Improved one-dimensional fin solutions, Heat Transf. Eng. 11 (1) (1989) 49-59.

[23] R.M. Cotta, Improved lumped-differential formulations in heat transfer, Invited Chapter, in: B. Sunden, M. Faghri (Eds.), Modelling of Engineering Heat Transfer Phenomena, Chap. 10, vol. 2, Heat Transfer Series, Computational Mechanics Publ., UK, 1998, pp. 293-325.

[24] L.B. Dantas, H.R.B. Orlande, R.M. Cotta, Improved lumped-differential formulations and hybrid solution methods for drying in porous media, Int. J Thermal Sci. 46 (9) (2007) 878-889.

[25] D.C. Knupp, C.P. Naveira-Cotta, J.V.C. Ayres, R.M. Cotta, H.R.B. Orlande, Identification of Thermophysical Properties of Heterogeneous Nanocomposites Via Integral Transforms, Bayesian Inference and Infrared Thermography, 7th Int Conf. on Inverse Problems in Engineering, ICIPE, Orlando, Florida, USA, May 4-6, 2011.

[26] J.E. Mark, Physical Properties of Polymers Handbook, Springer, New York, 2007

[27] T. Lewis, L.E. Nielsen, Dynamic mechanical properties of particulate-filled polymers, J. Appl. Polym. Sci. 14 (6) (1970) 1449-1471.

[28] J.H. Lienhard IV, J.H. Lienhard V, A Heat Transfer Textbook, Phlogiston Press, Cambridge, MA, US, 2008

[29] C.P. Naveira, M. Lachi, R.M. Cotta, J. Padet, Hybrid formulation and solution for transient conjugated conduction-external convection, Int. J. Heat Mass Transfer 52 (1-2) (2009) 112-123.

[30] C.P. Naveira-Cotta, M. Lachi, M. Rebay, R.M. Cotta, Experiments and simulations in transient conjugated conduction-convection-radiation, Heat Transfer Res. 43 (3) (2010) 209-231. 